\title{
Higher homotopy commutativity of small ring spectra
}

\author{
E. S. Devinatz ${ }^{1}$
}

Received: 13 January 2015 / Accepted: 15 March 2016 / Published online: 18 April 2016

(C) Tbilisi Centre for Mathematical Sciences 2016

\begin{abstract}
Let $X$ be a 2-local ring spectrum of type $n$ which is homotopy commutative up to a finite level of coherence (homotopy associativity is not assumed). We prove that, if $f: \Sigma^{k} X \rightarrow X$ is a $v_{n}$ self-map, there exists an $N$ such that, for all $m>0$, the cofiber $C\left(f^{m N}\right)$ of $f^{m N}$ has a ring spectrum structure extending that on $X$ which is homotopy commutative up to the same level of coherence. A strong analogue of this result holds at odd primes. We also discuss the relevance of this result to the problem of constructing explicit $v_{n+1}$ self-maps and provide a framework for extending our results to higher multiplicative structures including higher homotopy associativity.
\end{abstract}

Keywords Type $n$ finite spectra $\cdot v_{n}$ self-maps $\cdot$ Extended powers

\section{Mathematics Subject Classification 55P42}

\section{Introduction}

Let $X$ be a ring spectrum, by which we mean an object $X$ in the stable category together with morphisms $\mu: X \wedge X \rightarrow X$ and $\eta: \Sigma^{\infty} S^{0} \rightarrow X$ such that

$$
X=\Sigma^{\infty} S^{0} \wedge X \stackrel{\eta \wedge X}{\longrightarrow} X \wedge X \stackrel{\mu}{\longrightarrow} X
$$

Communicated by Mark Behrens.

$凶 \quad$ E. S. Devinatz

devinatz@math.washington.edu

1 Department of Mathematics, University of Washington, Seattle, WA 98195-4350, USA 
is the identity there. In particular, neither (homotopy) associativity nor commutativity is assumed; it is not even assumed that $\eta$ is a two-sided unit. In [2], we gave conditions on a self-map $f: \Sigma^{k} X \rightarrow X$ so that the cofiber $C(f)$ of $f$ is itself a ring spectrum and so that the inclusion $X \rightarrow C(f)$ is a map of ring spectra. We also observed that, if $X$ is in addition of type $n>0$ for a prime $p$ and if $g$ is a $v_{n}$ self-map, then nilpotence technology implies that there exists $N>0$ such that $g^{m N}$ satisfies the requisite hypotheses for all $m>0$. Hence $C\left(g^{m N}\right)$ is a ring spectrum of type $n+1$, and this process may be continued.

The result that a finite ring spectrum of type $n$ has a $v_{n}$ self-map such that the cofiber of any of its iterates is also a ring spectrum was one of the original conjectures now known collectively as the Ravenel conjectures ([12, Sect. 10]). (Of course, all of these conjectures are now theorems, with the exception of the Telescope conjecture, which remains open for $n>1$ ). The motivation for this conjecture came from the problem of - in modern terminology—constructing explicit $v_{n}$ self-maps. Indeed, if $X$ is a ring spectrum, then any map $\bar{f}: S^{k} \rightarrow X$ gives rise to the self-map

$$
\Sigma^{k} X=X \wedge S^{k} \stackrel{X \wedge \bar{f}}{\longrightarrow} X \wedge X \longrightarrow X
$$

which, of course, restricts to $\bar{f}$ on $S^{k}=\Sigma^{k} S^{0}$.

For example, recall how the $\beta$ family in $\pi_{*} S^{0}$ for $p \geq 5$ is constructed. Write $M\left(p^{i}\right)$ for the $\bmod \left(p^{i}\right)$ Moore spectrum and $M\left(p^{i_{0}}, v_{1}^{i_{1}}, \ldots, v_{n-1}^{i_{n-1}}\right)$ for a type $n$ spectrum obtained as the cofiber of a self map on $M\left(p^{i_{0}}, v_{1}^{i_{1}}, \ldots, v_{n-2}^{i_{n-2}}\right)$ which induces multiplication by $v_{n-1}^{i_{n-1}}$ on $B P$-homology. If $p \geq 3$, it follows from dimensional reasons in the Adams-Novikov spectral sequence that there exists a map

$$
\bar{v}_{2}: S^{2(p-1)} \rightarrow M\left(p, v_{1}\right) \equiv V(1)
$$

inducing multiplication by $v_{2}$ in $B P$-homology. If $p \geq 5, V(1)$ is a ring spectrum and thus the construction of 1.1 may be used to obtain a self-map $v_{2}: \Sigma^{2\left(p^{2}-1\right)} V(1) \rightarrow$ $V(1)$. Then $\beta_{t}$ is defined to be the composition

$$
\Sigma^{2 t\left(p^{2}-1\right)} S^{0} \longrightarrow \Sigma^{2 t\left(p^{2}-1\right)} V(1) \stackrel{v_{2}^{t}}{\longrightarrow} V(1) \longrightarrow S^{2 p},
$$

where the left map is the inclusion of the bottom cell and the right map is the collapse onto the top cell. On the other hand, if $p=3, V(1)$ is not a ring spectrum and in fact $M\left(3, v_{1}, v_{2}\right)$ does not exist. (Yet $M\left(3, v_{1}^{t}\right)$ is a ring spectrum for $t \geq 2$ [10]).

One might further ask whether these spectra have ring spectrum structures which are homotopy commutative and/or homotopy associative. Such issues were considered by Oka [11], who used his results to construct explicit $v_{n}$ self-maps.

Of course, Oka was working before the general results of Hopkins and Smith on $v_{n}$ self-maps [6] and so he limited his investigations to only certain type $n$ spectra for small values of $n$. However, his construction may be described in more general terms, and — at the risk of becoming overly technical—we feel that some readers may appreciate a synopsis. 
Suppose then that $\left(p^{i}, w_{1}, \ldots, w_{n-1}\right)$ is an invariant ideal in $B P_{*}$ with

$$
w_{i} \equiv v_{i}^{t_{i}} \quad \bmod \left(p, v_{1}, \ldots, v_{i-1}\right)
$$

for $1 \leq i \leq n$, and that $X$ is a homotopy commutative and homotopy associative finite ring spectrum with $B P_{*} X \approx B P_{*} /\left(p^{i}, w_{1}, \ldots, w_{n-1}\right)$ as algebras over $B P_{*}$. Assume that

$$
f_{n}: \Sigma^{2 t_{n}\left(p^{n}-1\right)} X \longrightarrow X
$$

is a self-map such that $B P_{*} f_{n}$ is multiplication by an element $w_{n} \in B P_{*}$ with

$$
w_{n} \equiv v_{n}^{t_{n}} \quad \bmod \left(p, v_{1}, \ldots, v_{n-1}\right)
$$

(Since any self-map of a spectrum with cyclic $B P$-homology is just multiplication by an element of $B P_{*}$, we will write

$$
\left.B P_{*} f_{n}=w_{n} \equiv v_{n}^{t_{n}} \bmod \left(p, v_{1}, \ldots, v_{n-1}\right) .\right)
$$

Let $d_{m}$ denote the composition

$$
C\left(f_{n}^{m}\right) \stackrel{\partial}{\longrightarrow} \Sigma^{2 m t_{n}\left(p^{n}-1\right)+1} X \stackrel{i}{\longrightarrow} \Sigma^{2 m t_{n}\left(p^{n}-1\right)+1} C\left(f_{n}^{m}\right)
$$

where $\partial$ and $i$ are the maps in the cofiber sequence induced by $f_{n}^{m}$. If $C\left(f_{n}^{m}\right)$ has the structure of a homotopy commutative and homotopy associative ring spectrum such that $d_{m}$ is a derivation, and if $f_{n+1, m}$ is a self-map of $C\left(f_{n}^{m}\right)$ with

$$
B P_{*} f_{n+1, m} \equiv v_{n+1}^{t} \quad \bmod \left(p, v_{1}, \ldots, v_{n}\right)
$$

then Oka observed that there exists a self-map $f_{n+1,2 m}$ of $C\left(f_{n}^{2 m}\right)$ with

$$
B P_{*} f_{n+1,2 m} \equiv v_{n+1}^{p^{i} t} \quad \bmod \left(p, v_{1}, \ldots, v_{n}\right)
$$

This procedure may of course be iterated-assuming $C\left(f_{n}^{m}\right)$ has the requisite multiplicative properties - to obtain explicit $v_{n+1}$ self-maps for an entire family of type $(n+1)$ spectra. Observe also that if $s \leq 2^{j}$, we may form the composition

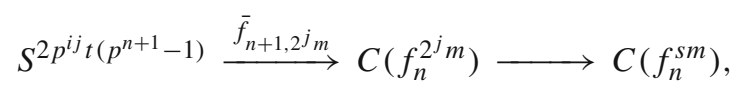

where the left map is the restriction of $f_{n+1,2^{j} m}$ to the unit and the right map is the evident projection. We may than use the process of 1.1 to extend this map to a self-map $f_{n+1, s m}$ of $C\left(f_{n}^{s m}\right)$ with

$$
B P_{*} f_{n+1, s m} \equiv v_{n+1}^{t p^{i j}} \quad \bmod \left(p, v_{1}, \ldots, v_{n}\right)
$$


Oka was able to show that the spectra $M\left(p, v_{1}^{s}\right)$ for $p \geq 5, s \geq 1$, and $M\left(p, v_{1}, v_{2}^{s}\right)$ for $p \geq 7,1 \leq s \leq[(p-2) / 3]$, have homotopy commutative and homotopy associative multiplications. However, the general question as to whether a ring spectrum of, say, the form $M\left(p^{i_{0}}, v_{1}^{i_{1}}, \ldots, v_{n}^{i_{n}}\right)$ has a homotopy commutative and homotopy associative multiplication is apparently quite subtle, even in the case $n=0$. Indeed, $M(2)$ is not a ring spectrum and $M(4)$ is a ring spectrum which is not homotopy commutative. If $i \geq 3, M\left(2^{i}\right)$ has a homotopy commutative multiplication which is also homotopy associative if $i \geq 4$. In this case, though, note that there is a $v_{0}$ self-map $f: S_{(2)}^{0} \rightarrow S_{(2)}^{0}$ such that $\bar{C}\left(f^{m}\right)$ has the desired multiplicative properties for all $m$.

One is naturally, then, led to ask whether the result of [2] can be refined to produce homotopy commutative or homotopy associative ring spectra or even ring spectra with higher multiplicative structures. In this paper, we will extend our previous result to obtain ring spectra commutative up to arbitrary finite homotopy. Although we have no immediate applications of higher multiplicative structures on these spectra, we will see that looking at higher homotopies clarifies the issues involved and that it is no harder to obtain results for higher commutativity than it is for just ordinary homotopy commutativity. However, the existence of a multiplication commutative up to higher homotopies imposes more and more stringent requirements on a (2-local) finite spectrum: we will prove in "Appendix 1" that, for each $i, M\left(2^{i}\right)$ is commutative up to only a finite number of higher homotopies. On the other hand, at odd primes, the issue of higher homotopy commutativity is essentially trivial (see Remark 1.2 and Sect. 4), although we will also prove in "Appendix 1" that, for each $i$, the $p$ fold multiplication on $M\left(p^{i}\right)$ is commutative up to only a finite number of higher homotopies as well.

We now make our notion of higher homotopy commutativity precise. Let $E$ be a free connected $\mathbb{Z} /(2)-C W$ complex, and let $X$ be an object in a suitable model for the stable category (we will say more about this below). An E-commutative ring spectrum structure on $X$ is a pair of maps

$$
\begin{aligned}
& \xi: E \ltimes \mathbb{Z} /(2)(X \wedge X) \rightarrow X \\
& \eta: \Sigma^{\infty} S^{0} \rightarrow X
\end{aligned}
$$

such that $X$ becomes a ring spectrum with unit $\eta$ and multiplication

$$
\mu: X \wedge X=\mathbb{Z} /(2) \ltimes_{\mathbb{Z} /(2)}(X \wedge X) \longrightarrow E \ltimes_{\mathbb{Z} /(2)}(X \wedge X) \stackrel{\xi}{\longrightarrow} X .
$$

Here $\mathbb{Z} /(2) \rightarrow E$ is any $\mathbb{Z} /(2)$-equivariant map; any two such maps will yield homotopic multiplications. In particular, $\mu$ is homotopy commutative. The space $E$ of course organizes information about the homotopy commutativity and all the higher homotopies involved. We can now state our main result.

Theorem 1.1 Let $X$ be a 2-local E-commutative ring spectrum of type $n$, E finite, and let $g: \Sigma^{|g|} X \rightarrow X$ be a $v_{n}$ self-map. (If $n=0$, we assume that $X=\Sigma^{\infty} S_{(2)}^{0}$; then $g$ will just be multiplication by a power of 2.) There exists $N>0$ such that, for any $m>0, C\left(g^{m N}\right)$ has an E-commutative ring spectrum structure extending that on $X$. 
Remark 1.2 If $p>2$, the strongest variant of this result holds. Namely, we will show (Propositions 4.2 and 4.4) that the $p$-local analogue of this theorem is true whenever $E=W$ is a contractible free $\mathbb{Z} /(2)-C W$ complex. In fact, any homotopy commutative ring spectrum structure on a connective $p$-local object $X$ extends uniquely to an $E$ commutative ring spectrum structure. This of course does not hold when $p=2$.

Remark 1.3 If $g: X \rightarrow X$ is a $v_{0}$ self-map, $X$ a spectrum of type 0 , then by nilpotence technology (see Theorem 2.1), some iterate of $g$ is just multiplication by $p^{i}$. Replacing $g$ by this iterate, it then follows that $C\left(g^{m}\right)=X \wedge M\left(p^{i m}\right)$. If $X$ is an $E$-commutative ring spectrum, then any $E$-commutative ring spectrum structure on $M\left(p^{i m}\right)$ extending the canonical such structure on $\Sigma^{\infty} S_{(p)}^{0}$ yields an $E$-commutative ring spectrum structure on $C\left(g^{m}\right)$ extending that on $X$.

In general, say that $X$ has a $j$-fold multiplication with unit if there exist maps $\mu: X^{(j)} \rightarrow X$ and $\eta: S^{0} \rightarrow X$ such that $\mu \circ\left(\eta^{(j-1)} \wedge X\right):\left(S^{0}\right)^{(j-1)} \wedge X \rightarrow X$ is the identity (in the stable category). If $E$ is a free connected $\Sigma_{j}-\mathrm{CW}$ complex, say that the $j$-fold multiplication $\mu$ extends over $E \ltimes_{\Sigma_{j}} X^{(j)}$ if there exists a map $\xi: E \ltimes_{\Sigma_{j}} X^{(j)} \rightarrow X$ such that the composition

$$
X^{(j)}=\Sigma_{j} \ltimes_{\Sigma_{j}} X^{(j)} \stackrel{\iota_{j}(X)}{\longrightarrow} E \ltimes_{\Sigma_{j}} X^{(j)} \stackrel{\xi}{\longrightarrow} X
$$

is $\mu$. As before, $\iota_{j}(X)$ is induced by any equivariant map $\Sigma_{j} \rightarrow E$. We will be most interested in the cases where $E=E \Sigma_{j}$, a free contractible $\Sigma_{j}$-complex (which we always assume to have a finite number of cells in each dimension), or $E=E \Sigma_{j}^{N}$, the $N$-skeleton of such a complex. The following result was alluded to earlier.

Theorem 1.4 For each prime $p$ and $i \geq 1$, there exists an $N$ such that $M\left(p^{i}\right)$ does not have a $p$-fold multiplication with unit extending over $E \Sigma_{p}^{N} \ltimes_{\Sigma_{p}} M\left(p^{i}\right)^{(p)}$.

Theorems 1.1 and 1.4 now suggest that, for a type $n$ spectrum $X$, the following sort of structure might be relevant. This structure might be thought of as an action in the stable category of a finite skeleton of an $E_{\infty}$ operad on $X$. In more detail, fix positive integers $M \geq 2$ and $k_{2}, \ldots, k_{n}$, and assume that there exist maps

$$
\begin{aligned}
& \eta: \Sigma^{\infty} S^{0} \rightarrow X \\
& \xi_{j}: E \Sigma_{j}^{k_{j}} \ltimes_{\Sigma_{j}} X^{(j)} \rightarrow X \quad 2 \leq j \leq M
\end{aligned}
$$

such that the diagrams in [1, Chapter 1, Definition 3.1] commute in the stable category when restricted to appropriate skeleta and appropriate values of $j$ and $k$. (Here $\xi_{0}=\eta$ and $\left.\xi_{1}=i d\right)$. Then, if $g: \Sigma^{|g|} X \rightarrow X$ is a $v_{n}$ self-map, one might ask whether there exists $N$-depending of course on $k_{2}, \ldots, k_{M}$-such that $C\left(g^{m N}\right)$ has the same structure for any positive integer $m$. Theorem 1.1 answers the question affirmatively for $M=2$, and in "Appendix 2" we will describe a framework for attacking the question in general.

Our use of extended powers necessitates working in a model for the stable category with good point-set properties including an associative, commutative, and unital smash 
product. Our choice for such a model is the category of $S$-modules of [5]. Although the point-set description of this category - most notably the smash product-is somewhat complicated, the advantage of it is that one can often deal with $S$-modules as one does with ordinary pointed topological spaces. Just as with spaces, each $S$-module is fibrant, and there is a good notion of CW objects. The stable category may then be regarded as the category whose objects are the CW $S$-modules and whose morphisms are the homotopy classes of $S$-module maps, defined in the evident way. We also use this analogy between $S$-modules and spaces in Sect. 5, where we employ standard identities involving space-level cofibers in the context of $S$-modules. For the less expert reader, we have included in "Appendix 3" a brief account of the construction of the category of $S$-modules as well as a sketch of how these sorts of basic results are obtained.

We are interested in $S$-modules with an action of $\Sigma_{j}$ (in the category of $S$-modules). If $Y$ is such a spectrum, and $E$ is a free $\Sigma_{j}$-CW complex, then

$$
E \ltimes \Sigma_{j} Y \equiv E_{+} \wedge_{\Sigma_{j}} Y
$$

where $E_{+} \wedge Y$ is just the smash product of $Y$ with the space $E_{+}$. We will write $E_{+} \wedge_{\Sigma_{j}} Y$ as $e_{j}(Y)$ or as $e_{E, j}(Y)$ if $E$ needs to be specified. If $f: Y \rightarrow Z$ is a $\Sigma_{j}$-equivariant map of $S$-modules, we will write $e_{j}(f)$ for $E_{+} \wedge_{\Sigma_{j}} f$. Since we will only be considering $j=2$ for most of the paper, we will write $e(Y)$ or $e_{E}(Y)$ or $e(f)$ for $e_{2}(Y)$ or $e_{E, 2}(Y)$ or $e_{2}(f)$. In particular, if $X$ is an $S$-module, then the $S$-module $X^{(j)}$ is acted upon by $\Sigma_{j}$ via permutations, and we can form $E \ltimes \Sigma_{j} X^{(j)}$.

There is one more fact about smash products of $S$-modules worth mentioning now. If $A$ is a pointed space and $X$ is an $S$-module, then $A \wedge X$ is defined in the obvious way and is naturally isomorphic to $\Sigma^{\infty} A \wedge X$. We will from now on-except as noted in "Appendix 1"- -always write the suspension spectrum of a pointed space $A$ as $\Sigma^{\infty} A$, leaving the notation $A$ for the space itself. However, even if $A$ has the homotopy type of a based CW-complex, $\Sigma^{\infty} A$ has the homotopy type of a CW $S$-module if and only if it is contractible (see [4]). We thus write $\Gamma \Sigma^{\infty} A$ for a $\mathrm{CW} S$-module weakly homotopy equivalent to $\Sigma^{\infty} A$.

Here now is the organization of this paper. We begin by recalling the needed nilpotence technology and obtain some general consequences of this technology. This is carried out in Sect. 2. In Sect. 3, we establish some properties of the twisted halfsmash product construction; in particular, with $j=2$, we construct a stable map $\langle f\rangle: e_{E}(X) \rightarrow e_{E}(Y)$ whenever $f$ is a (non-equivariant) map from $X$ to $Y$. This map may be thought of as $e_{E}$ applied to the stable map $f+\sigma f \sigma: X \rightarrow Y$, where $\sigma: X \rightarrow X$ and $\sigma: Y \rightarrow Y$ both denote the action of the nontrivial element $\sigma$ of $\mathbb{Z} /(2)$. With these preliminaries, the proof of our version of Theorem 1.1 for $p>2$ is now easy and is carried out in Sect. 4. The rest of the paper is then devoted to the proof of Theorem 1.1. An outline is given in Sect. 5 with the omitted details provided in Sects. 6, 7, 8 and 9. The main issue here is showing that the homotopies making certain squares homotopy commutative are in a certain sense compatible, so that the induced maps on cofibers are also compatible, again in an appropriate sense.

As indicated earlier, there are three Appendices. In the first, we give a short proof of 1.4 , and in the second, more speculative, Appendix, we describe our proposed strategy 
for proving the above far-reaching generalization of the main result of this paper. The third Appendix recalls some of the constructions needed to work with $S$-modules.

\section{Nilpotence technology}

Let $X$ be a finite $p$-local CW $S$-module which is $K(n-1)_{*}$-acyclic. A $v_{n}$ self-map is a map $f: \Sigma^{k} X \rightarrow X$ such that $K(n)_{*} f$ is an isomorphism and $K(m)_{*} f$ is nilpotent for $m \neq n$. If $n=0$, we make the additional assumption that $H \mathbb{Q}_{*} f$, the map induced by $f$ on ordinary rational homology, is multiplication by $p^{i}$ for some $i$. (Recall that $K(0)$ is just $H \mathbb{Q}$ ). The following result is what we will in this paper refer to as nilpotence technology.

\section{Theorem 2.1 [6]}

(i) If $f: \Sigma^{k} X \rightarrow X$ and $g: \Sigma^{l} X \rightarrow X$ are $v_{n}$ self-maps, then there exist $r$ and $s$ such that $f^{r}$ is homotopic to $g^{s}$.

(ii) If $f: \Sigma^{k} X \rightarrow X$ and $g: \Sigma^{l} Y \rightarrow Y$ are $v_{n}$ self-maps, then there exist $r$ and $s$ such that $h \circ f^{r} \simeq g^{s} \circ h$ for any $h \in[X, Y]_{*}$. In particular, some iterate of $f$ is in the center of the ring $[X, X]_{*}$.

Remark 2.2 Of course, part i follows from part ii but Hopkins and Smith prove ii as a consequence of i. Indeed, let $D X$ denote the ( $p$-local) Spanier-Whitehead dual of $X$ and consider $Y \wedge D X$, which is naturally equivalent to the stable category function object of maps from $X$ to $Y$. Since $g \wedge D X$ and $Y \wedge D f$ are both $v_{n}$ self-maps, part i implies that $Y \wedge D f^{r} \simeq g^{s} \wedge D X$ for some $r$ and $s$. It now follows formally from the adjunction property of the function object that $f^{r}$ and $g^{s}$ have the property given in part ii.

In this paper we will use several general consequences of the above result. Our first consequence is a generalization of a key technical piece of [2].

Proposition 2.3 Suppose that $g: \Sigma^{k} Y \rightarrow Y$ and $h: \Sigma^{k} Z \rightarrow Z$ are $v_{n}$ self-maps with $k$ even (and induce multiplication by the same power of $p$ on rational homology if $n=0)$. Then there exists $N$ such that, for any $m>0$,

$$
h^{m N} \wedge C\left(g^{m N}\right): \Sigma^{m N k} Z \wedge C\left(g^{m N}\right) \rightarrow Z \wedge C\left(g^{m N}\right)
$$

is trivial.

This result follows by nilpotence technology from the following.

Lemma 2.4 Let $Y$ and $Z$ be $C W$-modules, and let $g: \Sigma^{k} Y \rightarrow Y$ and $h: \Sigma^{k} Z \rightarrow Z$ with $k$ even. Suppose that $h \wedge Y$ is central in the ring $[Z \wedge Y, Z \wedge Y]_{*}$ and is homotopic to $Z \wedge g$. Then

$$
h^{2} \wedge C\left(g^{2}\right): \Sigma^{2 k} Z \wedge C\left(g^{2}\right) \rightarrow Z \wedge C\left(g^{2}\right)
$$

is trivial. 
Proof First mimic the proof of Lemma 4 of [2] to show that, if $h \wedge Y \simeq Z \wedge g$, there exists a map $\Sigma^{k} Z \wedge \Sigma^{k+1} Y \rightarrow Z \wedge Y$ such that the diagram

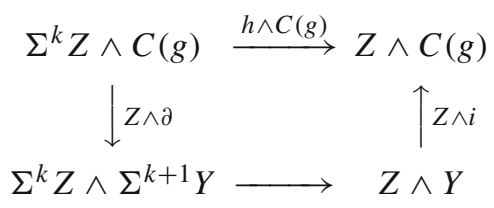

is homotopy commutative, where $\partial$ and $i$ are the maps in the evident cofibration sequence. Then mimic the proof of Lemma 5 of [2] to show that if, in addition, $h \wedge Y$ is central, then $h^{2} \wedge C\left(g^{2}\right)$ is trivial.

Our next consequence will give us some point-set control in dealing with homotopies between $v_{n}$ self-maps. Let us first introduce some notation.

As usual, if $X$ is a topological space and $x_{0} \in X$ we write $c_{x_{0}}$ for the constant loop at $x_{0}$. We also write $\bar{\omega}$ for the reverse of a path $\omega, *$ for the operation of path multiplication, and $\simeq p$ for path homotopy.

If $X$ and $Y$ are $S$-modules (resp. pointed spaces), let $\operatorname{Map}^{S}(X, Y)(\operatorname{resp} . \operatorname{Map}(X, Y))$ denote the topological space of $S$-module maps (resp. pointed maps) from $X$ to $Y$. If $f: X \rightarrow Y, \operatorname{Map}_{f}^{S}(X, Y)$ (resp. $\operatorname{Map}_{f}(X, Y)$ ) will denote the path component of $\operatorname{Map}^{S}(X, Y)(\operatorname{resp} . \operatorname{Map}(X, Y))$ containing $f$. If this space is to be regarded as a based space, $f$ will be its basepoint. In this context, we will denote the trivial map by $*$, so that, for example, $\operatorname{Map}_{*}^{S}(X, Y)$ denotes the path component of $\operatorname{Map}^{S}(X, Y)$ containing the trivial map.

Suppose now that $Z$ is a topological space and $f: Z \rightarrow \operatorname{Map}^{S}(X, Y), g: Z \rightarrow$ $\operatorname{Map}^{S}(W, X)$. We will write $f \circ g$ for the map $Z \rightarrow \operatorname{Map}^{S}(W, Y)$ which sends $Z$ to $f(z) \circ g(z)$. If $f$ or $g$ is just an element of $\operatorname{Map}^{S}(X, Y)$ or $\operatorname{Map}^{S}(W, X)$, then $f \circ g: Z \rightarrow \operatorname{Map}^{S}(W, Y)$ will be defined by regarding $f$ or $g$ to be the constant map sending $Z$ to $f$ or $g$. If $f: Z \rightarrow \operatorname{Map}^{S}\left(\Sigma^{k} X, X\right)$, then $f^{(N)}: Z \rightarrow \operatorname{Map}^{S}\left(\Sigma^{k N} X, X\right)$ denotes the map $f \circ \Sigma^{k} f \circ \cdots \circ \Sigma^{k(N-1)} f$. We will also use this notation when $W$, $X$, and $Y$ are pointed topological spaces and we are dealing with $\operatorname{Map}(\quad, \quad)$.

Proposition 2.5 Let $X$ be a $C W S$-module, and suppose that $f: \Sigma^{k} X \rightarrow X$ is central in $[X, X]_{*}$. If $h \in \pi_{1}\left(\operatorname{Map}_{f}^{S}\left(\Sigma^{k} X, X\right)\right)$, then $h \circ \Sigma^{k} \bar{h} \in \pi_{1}\left(\operatorname{Map}_{f^{2}}^{S}\left(\Sigma^{2 k} X, X\right)\right)$ is trivial.

Proof First observe that

$$
h \circ \Sigma^{k} \bar{h} \simeq p\left(f \circ \Sigma^{k} \bar{h}\right) *\left(h \circ \Sigma^{k} f\right) .
$$

The centrality of $f$ implies that $g \circ\left(S_{+}^{1} \wedge f\right)=f \circ g$ in $\left[S_{+}^{1} \wedge X, X\right]_{*}$ for any $g \in\left[S_{+}^{1} \wedge X, X\right]_{*}$. Taking adjoints, this means that $f \circ \Sigma^{k} h: S^{1} \rightarrow \operatorname{Map}^{S}\left(\Sigma^{2 k} X, X\right)$ is freely homotopic to $h \circ \Sigma^{k} f$. Since each path component of $\operatorname{Map}^{S}\left(\Sigma^{2 k} X, X\right)$ is simple-if $k>0$, this follows because $\operatorname{Map}^{S}\left(\Sigma^{2 k} X, X\right)$ is an $H$-group, and if $k=0$, this follows from Remark 2.6 below- $f \circ \Sigma^{k} h \simeq{ }_{p} h \circ \Sigma^{k} f$ and the result is immediate from 2.1. 
Remark 2.6 In general, if $Y$ is a $C W S$-module and $Z$ is any $S$-module, $\operatorname{Map}^{S}(Y, Z)$ is weakly homotopy equivalent to the $H$-group $\operatorname{Map}^{S}(Y, \Omega \Sigma Z)$ [5, I, Corollary 6.3]. Hence each path component of $\operatorname{Map}^{S}(Y, Z)$ is simple.

Remark 2.7 Elmendorf has proved [4] that if $Y$ and $Z$ are pointed spaces, then $\operatorname{Map}(Y, Z) \approx \operatorname{Map}^{S}\left(\Sigma^{\infty} Y, \Sigma^{\infty} Z\right)$. Remark 2.6 is therefore not true without some sort of assumption on the domain $S$-module.

Remark 2.8 With the hypotheses of Proposition 2.5, the same sort of argument may be used to prove that

$$
h^{(N)} \simeq_{p} N\left(f^{N-1} \circ \Sigma^{k N} h\right)=f^{N-1} \circ \Sigma^{k N} N h .
$$

Consequently, if $N \pi_{1}\left(\operatorname{Map}_{f}^{S}\left(\Sigma^{k} X, X\right)\right)$ is trivial, then so is $h^{(N)}$. Since $\operatorname{Map}_{f}^{S}\left(\Sigma^{k} X, X\right)$ is weakly homotopy equivalent to $\operatorname{Map}_{*}^{S}\left(\Sigma^{k} X, X\right)$, this is the case if and only if $N[X, X]_{k+1}=0$.

These considerations imply the following result: suppose that $X$ is a $K(n-1)_{*^{-}}$ acyclic finite CW $S$-module and that $g: \Sigma^{k} X \rightarrow X$ is a $v_{n}$ self-map (if $n=0$, we assume that $\left.X=\Gamma \Sigma^{\infty} S_{(p)}^{0}\right)$. If $h \in \pi_{1}\left(\operatorname{Map}_{g}^{S}\left(\Sigma^{k} X, X\right)\right)$, then there exists $N$ such that $h^{(N)} \simeq c_{g^{N}}$.

\section{Extended powers}

In this section, we establish some properties of the extended power construction, and, more generally, the construction $e(Y) \equiv E_{+} \wedge_{\mathbb{Z} /(2)} Y$ for $Y$ an $S$-module equipped with a $\mathbb{Z} /(2)$ action. Normally, the actions on our $S$-modules will come from the switch map, and, unless explicitly mentioned otherwise, this will be the presumed action whenever it makes sense. For example, if we write $Y \wedge X \vee X \wedge Y$, the action by the nontrivial element of $\mathbb{Z} /(2)$ is taken to be the map which interchanges the summands and switches the factors.

We will also need to consider the action of $\mathbb{Z} /(2)$ on various suspensions. Normally, if $Y$ is an $S$-module and we write $e\left(\Sigma^{j} Y\right)$, we are giving $\Sigma^{j} S^{0}$ the trivial $\mathbb{Z} /(2)$ action. However, if we write $e\left(\Sigma^{j} X \wedge \Sigma^{j} X\right), \mathbb{Z} /(2)$ is assumed to act by switching the factor $\Sigma^{j} X$. If we write $e\left(\Sigma_{-}^{j} Y\right)$, then $\mathbb{Z} /(2)$ acts on $\Sigma_{-}^{j} S^{0}$ by sending $\left[t_{1}, \ldots, t_{j}\right]$ to $\left[-t_{1}, \ldots,-t_{j}\right]$, where the suspension coordinates are taken to be in $[-1,1]$.

Finally, we will be shuffling suspension coordinates in our construction of various maps. When it's not clear how we are doing this, we will add subscripts to the suspension notation. For example, we might write something like $\Sigma_{1}^{k} \Sigma_{2}^{k}(X \wedge X)=$ $\Sigma_{1}^{k} X \wedge \Sigma_{2}^{k} X$.

The next result gives us what we will need about suspensions in extended powers. It is a version of an observation of Nishida [9].

Proposition 3.1 Let $E$ be a finite free $\mathbb{Z} /(2)-C W$ complex. Then there exists an $M$ (depending on $E$ ) such that, if $X$ is any $C W S$-module, $\Sigma^{2 M} e(X \wedge X)$ is homotopy equivalent to $e\left(\Sigma^{M} X \wedge \Sigma^{M} X\right)$. This equivalence is natural in $X$ in the stable category. 
The main ingredient in the proof is the fact that the canonical line bundle over a finite dimensional real projective space has finite order in reduced $K$-theory. Before getting to that, however, we need to recall a little about the relationship between the various $\mathbb{Z} /(2)$ actions on suspensions.

Let $\mathbb{R}$ denote the real line with trivial $\mathbb{Z} /(2)$ action, let $\mathbb{R}_{\text {- denote the real line with }}$ $\mathbb{Z} /(2)$ action given by $\sigma(t)=-t$, and let $(\mathbb{R} \oplus \mathbb{R})_{\text {reg }}$ denote the regular representation; i.e. $\sigma(s, t)=(t, s)$. There is an equivariant linear isomorphism $(\mathbb{R} \oplus \mathbb{R})_{\text {reg }} \rightarrow \mathbb{R} \oplus \mathbb{R}_{-}$ given by sending $(s, t)$ to $(s+t, s-t)$.

Now let $S(V)$ denote the one point compactification of the finite dimensional vector space $V$, and give $S(V)$ the basepoint $\infty$, the point at infinity. If $V$ is a representation of $\mathbb{Z} /(2)$, then $\mathbb{Z} /(2)$ acts on $S(V)$ via its action on $V$ and by fixing $\infty$. The above remarks then show that there are equivariant homeomorphisms

$$
\Sigma^{j} S^{0} \wedge \Sigma^{j} S^{0} \approx S\left((\mathbb{R} \oplus \mathbb{R})_{\mathrm{reg}}^{j}\right) \approx S\left(\mathbb{R}^{j} \oplus \mathbb{R}_{-}^{j}\right) \equiv \Sigma^{j} \Sigma_{-}^{j} S^{0}
$$

The next lemma is an alternate way to express the finite order of the canonical line bundle over $\mathbb{R} P^{m}$, which will prove convenient to us.

Lemma 3.2 Let $a_{m}$ denote the order of the canonical line bundle $\gamma_{m}$ in $\widetilde{K O}\left(\mathbb{R} P^{m}\right)$ and suppose that $a_{m} \mid l$. Then for $N$ sufficiently large, there exists a map $G: S^{m} \rightarrow$ $S O(l+N)$ such that $G(z)(u, v)=G(-z)(-u, v)$ for all $z \in S^{m}, u \in \mathbb{R}^{l}$, and $v \in \mathbb{R}^{N}$. If $a_{m+1} \mid l$, then for $N$ sufficiently large, $G$ may be chosen to be homotopic to a constant map.

Proof Let $\varepsilon^{j}$ denote the trivial $j$-plane bundle. The total space of the bundle $l \gamma_{m} \oplus \varepsilon^{N}$ is just $S^{m} \times \mathbb{Z / ( 2 )}\left(\mathbb{R}_{-}^{l} \oplus \mathbb{R}^{N}\right)$. But for $N$ sufficiently large, $l \gamma_{m} \oplus \varepsilon^{N} \approx \varepsilon^{N+l}$; this implies that there is a homeomorphism

$$
S^{m} \times_{\mathbb{Z} /(2)}\left(\mathbb{R}_{-}^{l} \oplus \mathbb{R}^{N}\right) \rightarrow S^{m} \times_{\mathbb{Z} /(2)} \mathbb{R}^{N+l}
$$

which is an isometry on each fiber. This homeomorphism sends $(z, u, v)$ to $(z, G(z)(u, v))$, where $G(z) \in O(N+l)$. Since $S O(N+l)$ is a component, we may assume that $G(z) \in S O(N+l)$. $G$ clearly satisfies the desired requirements.

If $a_{m+1} \mid l$, then for $N$ sufficiently large, $l \gamma_{m+1} \oplus \varepsilon^{N} \approx \varepsilon^{N+l}$, so we get a map $G^{\prime}: S^{m+1} \rightarrow S O(l+N)$ as above. Now take $G=G^{\prime} \mid S^{m}$. This map is homotopic to the constant map.

Proof of Proposition $3.1 E$ is the total space of a principal $\mathbb{Z} /(2)$-bundle; hence there exists an equivariant map $\theta: E \rightarrow S^{\infty}$. Since $E$ is finite, the image of $\theta$ lies in $S^{m}$ for some $m$, and we write $\theta: E \rightarrow S^{m}$.

Let $M=a_{m}$ and choose $N>M$ so that the conclusion of the preceding lemma holds with $l=a_{m}$. We then have an isomorphism

$$
E_{+} \wedge \mathbb{Z} /(2)\left[S\left(\mathbb{R}_{-}^{M} \oplus \mathbb{R}^{N}\right) \wedge X \wedge X\right] \rightarrow E_{+} \wedge_{\mathbb{Z} /(2)}\left[S\left(\mathbb{R}^{N+M}\right) \wedge X \wedge X\right]
$$

given by sending $(z, u, v, x)$ to $(z, G(\theta(z))(u, v), x)$ for $z \in E, u \in \mathbb{R}^{M}, v \in \mathbb{R}^{N}$, and $x \in X \wedge X$. (Note that $S O(j)$ extends to an action on $S\left(\mathbb{R}^{j}\right)$ by always mapping $\infty$ to itself). But 


$$
E_{+} \wedge \mathbb{Z} /(2)\left[S\left(\mathbb{R}^{N+M}\right) \wedge X \wedge X\right] \approx \Sigma^{N-M} \Sigma^{2 M} e(X \wedge X),
$$

and by 3.1 ,

$$
E_{+} \wedge \mathbb{Z} /(2)_{S}\left[S\left(\mathbb{R}_{-}^{M} \oplus \mathbb{R}^{N}\right] \wedge X \wedge X\right] \approx \Sigma^{N-M} e\left(\Sigma^{M} X \wedge \Sigma^{M} X\right)
$$

This completes the proof.

We next turn to the construction and properties of the stable map $\langle f\rangle: e(X) \rightarrow e(Y)$ alluded to in the Introduction; this map will play a key role in what follows.

Begin by recalling the space $C_{j}(2)$ of two little cubes in $\mathbb{R}^{j}$, as described in [8], for example. For our purposes, it will be the space of ordered pairs of nonoverlapping $j$-dimensional "cubes" in $[-1,1]^{j}$, with $\mathbb{Z} /(2)$ acting by switching the elements of the ordered pairs. By a $j$-dimensional cube, we mean a set of the form $\left[a_{1}, b_{1}\right] \times$ $\cdots \times\left[a_{j}, b_{j}\right]$ with $a_{i}<b_{i}$ for all $i$. There are inclusions $C_{j}(2) \rightarrow C_{j+1}(2)$ given by sending a cube $\left[a_{1}, b_{1}\right] \times \cdots \times\left[a_{j}, b_{j}\right]$ to the cube $\left[a_{1}, b_{1}\right] \times \cdots \times\left[a_{j}, b_{j}\right] \times[-1,1]$, and we set $C_{\infty}(2)=\lim _{\rightarrow} C_{j}(2) . C_{\infty}(2)$ is the total space of a universal principal $\mathbb{Z} /(2)$ bundle; this implies that if $E$ is a free $\mathbb{Z} /(2)$-complex, then there exists an equivariant map $E \rightarrow C_{\infty}$ (2), unique up to equivariant homotopy. If $E$ is in addition finite, then this map factors through $C_{N}$ (2) for $N$ sufficiently large, and two such maps are equivariantly homotopic as maps into $C_{N}(2)$, again for $N$ sufficiently large.

If $c=\left[a_{1}, b_{1}\right] \times \cdots \times\left[a_{j}, b_{j}\right]$, let us also write $c$ for the map $\prod_{i=1}^{j}\left[a_{i}, b_{i}\right] \rightarrow$ $[-1,1]^{j}$ which is the product of the increasing affine linear homeomorphisms sending $\left[a_{i}, b_{i}\right]$ to $[-1,1]$. As we will throughout the paper, regard the suspension coordinates as lying in $[-1,1]$, so that the space $\Sigma^{j} S^{0}$ is the quotient $[-1,1]^{j} / \partial\left([-1,1]^{j}\right)$. The cube $c$ can now also be regarded as a map

$$
c: \Sigma^{j} S^{0} \rightarrow \Sigma^{j} S^{0}
$$

defined by

$$
c\left[t_{1}, \ldots, t_{j}\right]= \begin{cases}{\left[c\left(t_{1}, \ldots, t_{j}\right)\right]} & \left(t_{1}, \ldots, t_{j}\right) \in c \\ * & \text { ow }\end{cases}
$$

Construction 3.3 Let $E$ be a finite free $\mathbb{Z} /(2)-C W$ complex and let $\iota: E \rightarrow C_{N}(2)$ be equivariant. Write $\iota(z)=\left(\iota_{1}(z), \iota_{2}(z)\right)$, where each $\iota_{i}(z)$ is a cube in $[-1,1]^{N}$. Suppose that $X$ and $Y$ are $C W S$-modules, each with a cellular $\mathbb{Z} /(2)$ action; the action on each spectrum by the nontrivial element of $\mathbb{Z} /(2)$ will be denoted by $\sigma$. If $f: X \rightarrow Y$ is a (not necessarily equivariant) map of $S$-modules, define

$$
\langle f\rangle_{\iota}: \Sigma_{R}^{N} e_{E}(X) \rightarrow \Sigma_{R}^{N} e_{E}(Y)
$$


by

$$
\langle f\rangle_{\iota}\left[z, x, t_{1}, \ldots, t_{N}\right]= \begin{cases}{\left[z, f(x), \iota_{1}(z)\left[t_{1}, \ldots, t_{n}\right]\right]} & {\left[t_{1}, \ldots, t_{n}\right] \in \iota_{1}(z)} \\ {\left[z, \sigma f \sigma(x), \iota_{2}(z)\left[t_{1}, \ldots, t_{n}\right]\right]} & {\left[t_{1}, \ldots, t_{n}\right] \in \iota_{2}(z)} \\ * & \text { ow }\end{cases}
$$

where $z \in E, x \in X$, and $\left[t_{1}, \ldots, t_{N}\right] \in \Sigma^{N} S^{0}$. The subscript $R$ here indicates that the suspension coordinates are to be written on the right.

Remark 3.4 If $\iota^{\prime}: E \rightarrow C_{N^{\prime}}(2)$ is another equivariant map, then there exists $M$ such that

$$
\iota: E \rightarrow C_{N}(2) \hookrightarrow C_{M}(2)
$$

and

$$
\iota^{\prime}: E \rightarrow C_{N^{\prime}}(2) \hookrightarrow C_{M}(2)
$$

are equivariantly homotopic. It then follows that $\Sigma_{R}^{M-N}\langle f\rangle_{\iota}$ is homotopic to $\Sigma_{R}^{M-N^{\prime}}\langle f\rangle_{\iota^{\prime}}$. Although this observation is reassuring, we shall in this paper be fixing our map $E \rightarrow C_{\infty}(2)$.

In this paper, we will often need to work at the point-set level of spectra and $S$ modules and therefore must use $\langle f\rangle_{\iota}$, instead of the stable map $e_{E}(X) \rightarrow e_{E}(Y)$ that it induces. We will, however, mostly delete the suspensions from the notation, as they usually just add clutter to already cluttered diagrams. We will also mostly delete the subscript $\iota$, since it will be fixed throughout the paper.

There is only one place in this paper-the proof of the odd prime analogue of Theorem 1.1-where we will need a construction of $\langle f\rangle_{\iota}$ valid for $E$ an infinite complex. In this situation, the map is only defined in the stable category, but that is good enough.

Construction 3.5 Suppose that $E$ is a free $\mathbb{Z} /(2)-C W$ complex and that $\iota: E \rightarrow$ $C_{\infty}(2)$ is equivariant. Consider the collection $\left\{E_{\alpha}\right\}$ of finite $\mathbb{Z} /(2)$-subcomplexes, ordered by inclusion, and write

$$
\left.\iota_{\alpha} \equiv \iota\right|_{E_{\alpha}}: E_{\alpha} \rightarrow C_{N_{\alpha}}(2)
$$

with $N_{\alpha}$ as small as possible. Let $f: X \rightarrow Y$ be as in Construction 3.3. We then have a commutative diagram

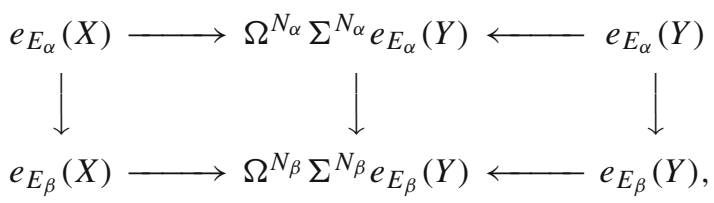


where the left horizontal maps are the adjoints of $\langle f\rangle_{\iota_{\alpha}}$ and $\langle f\rangle_{\iota_{\beta}}$, and the right horizontal maps are weak equivalences. Passing to homotopy colimits yields a stable map $\langle f\rangle_{\iota}: e_{E}(X) \rightarrow e_{E}(Y)$. Moreover, different choices of ı yield homotopic maps.

Constructions 3.3 and 3.5 have evident naturality properties and behave well with respect to homotopies. We will also single out the following property, which requires us to work with certain prespectra.

Say that a prespectrum $X$ is a pre $S$-module if its spectrification $L X$ is provided with an $\mathscr{L}$-algebra structure, so that $\Sigma^{\infty} S^{0} \wedge \mathscr{L} L X$ is an $S$-module. A map $f$ : $X \rightarrow Y$ between pre $S$-modules is a pre $S$-module map if $L f: L X \rightarrow L Y$ is an $\mathscr{L}$-algebra map, so that $\Sigma^{\infty} S^{0} \wedge \mathscr{L} L f$ is an $S$-module map. If $\mathbb{Z} /(2)$ acts on $X$ via pre $S$-module maps, then $X$ is called a pre $S$-module with $\mathbb{Z} /(2)$ action. Observe that, if $X$ is a prespectrum acted upon by $\mathbb{Z} /(2)$, then we may certainly form the prespectrum $E_{+} \wedge_{\mathbb{Z} /(2)} X \equiv e_{E}(X)$. If $X$ is a pre- $S$-module with $\mathbb{Z} /(2)$ action, then $\Sigma^{\infty} S^{0} \wedge \mathscr{L} L e_{E}(X)$ is naturally isomorphic to $e_{E}\left(\Sigma^{\infty} S^{0} \wedge \mathscr{L} L X\right)$. Finally, we will tacitly assume that $\Sigma^{\infty} S^{0} \wedge \mathscr{L} L X$ is a $C W S$-module and that the induced $\mathbb{Z} /(2)$ action is cellular whenever we speak of a pre $S$-module with $\mathbb{Z} /(2)$ action $X$.

Lemma 3.6 Let $X$ and $Y$ be pre $S$-modules with $\mathbb{Z} /(2)$ action, and give the pre $S$ module $Y \vee Y$ the $\mathbb{Z} /(2)$ action given by $\sigma(y, *)=(*, \sigma y)$ and $\sigma(*, y)=(\sigma y, *)$. Let $f: X \rightarrow Y \vee Y$ be an equivariant pre $S$-module map, and let $\Delta: Y \vee Y \rightarrow Y \vee Y$ denote the map which is the identity on the first summand and trivial on the second. If $E$ is a finite free $\mathbb{Z} /(2)-C W$ complex and $\iota: E \rightarrow C_{N}(2)$, then $\Sigma_{R}^{N} e_{E}(f)$ is homotopic to $\langle\Delta \circ f\rangle_{l}$.

Proof If $c=\prod_{i=1}^{N}\left[a_{i}, b_{i}\right]$ is an $N$-dimensional cube in $[-1,1]^{N}$ and $0 \leq t \leq 1$, write $(c, t)$ for the cube $\prod_{i=1}^{N}\left[(1-t) a_{i}-t,(1-t) b_{i}+t\right]$. Also write $f_{i}=\pi_{i} \circ f$, where $\pi_{i}: Y \vee Y \rightarrow Y$ is the projection onto the $i$ th summand.

Define a map

$$
\Sigma_{R}^{N} e_{E}(X) \wedge I_{+} \rightarrow \Sigma_{R}^{N} e_{E}(Y \vee Y)=E_{+} \wedge \mathbb{Z} /(2)\left(\Sigma^{N} Y \vee \Sigma^{N} Y\right)
$$

by sending $\left[z, x, t_{1}, \ldots, t_{N}, t\right]$ to

$$
\left[z,\left[\left(\iota_{1}(z), t\right)\left(t_{1}, \ldots, t_{N}\right), f_{1}(x)\right],\left[\left(\iota_{2}(z), t\right)\left(t_{1}, \ldots, t_{N}\right), f_{2}(x)\right]\right]
$$

where $z \in E, x \in X,\left[t_{1}, \ldots, t_{N}\right] \in \Sigma^{N} S^{0}$, and $t \in I$. This formula might require some unpacking: $\left[\left(\iota_{1}(z), t\right)\left(t_{1}, \ldots, t_{N}\right)\right]$ and $\left[\left(\iota_{2}(z), t\right)\left(t_{1}, \ldots, t_{N}\right)\right]$ are in $\Sigma^{N} S^{0}$, so

$$
\left(\left[\left(\iota_{1}(z), t\right)\left(t_{1}, \ldots, t_{N}\right), f_{1}(x)\right],\left[\left(\iota_{2}(z), t\right)\left(t_{1}, \ldots, t_{N}\right), f_{2}(x)\right]\right) \in \Sigma^{N} Y \times \Sigma^{N} Y .
$$

But for each $x$, either $f_{1}(x)=*$ or $f_{2}(x)=*$, so the above element is actually in $\Sigma^{N} Y \vee \Sigma^{N} Y$. It is now easy to check that this map is a homotopy from $\langle\Delta \circ f\rangle_{\text {, }}$ to $\Sigma_{R}^{N} e_{E}(f)$.

Remark 3.7 If $E$ is infinite and $\iota: E \rightarrow C_{\infty}$ (2), the above proof can be easily modified to show that $\langle\Delta \circ f\rangle_{\iota}=e_{E}(f)$ in the stable category. 


\section{The odd prime case}

We begin this section by showing how the main result of [2] can be refined to include two-sided units. This does not require working at an odd prime.

Lemma 4.1 Let $X$ be a ring spectrum with a two-sided unit, and let $f: \Sigma^{k} X \rightarrow X$ with $k$ even. Suppose that

(i) The map $f \wedge X: \Sigma^{k} X \wedge X \rightarrow X \wedge X$ is in the center of the ring $[X \wedge X, X \wedge X]_{*}$.

(ii) The diagram

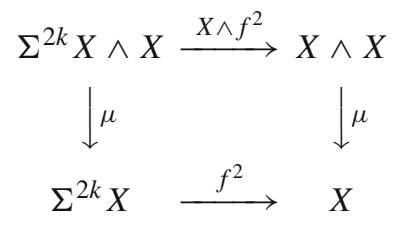

commutes (in the stable category).

(iii) $(X \wedge i)^{*}:\left[X \wedge C\left(f^{2}\right), C\left(f^{2}\right)\right]_{2 k+1} \rightarrow\left[X \wedge X, C\left(f^{2}\right)\right]_{2 k+1}$ is surjective, where $i: X \rightarrow C\left(f^{2}\right)$ denotes the usual inclusion.

Then $C\left(f^{2}\right)$ has the structure of a ring spectrum with a two-sided unit such that $i: X \rightarrow C\left(f^{2}\right)$ is a map of ring spectra.

Proof By Theorem 1 of [2], properties (i) and (ii) imply that $C\left(f^{2}\right)$ has the structure of a ring spectrum extending that on $X$. Property (iii) then implies (see [10, Discussion above Theorem 1.5]) that this structure can be chosen so that its unit is two-sided.

It may not be clear how condition (iii) relates to $v_{n}$ self-maps, but this can be dealt with. First observe that, if $X$ is $p$-locally finite,

$$
(X \wedge i)^{*}:\left[X \wedge C\left(f^{2}\right), C\left(f^{2}\right)\right]_{*} \rightarrow\left[X \wedge X, C\left(f^{2}\right)\right]_{*}
$$

is surjective provided that $X \wedge D C\left(f^{2}\right) \rightarrow C\left(f^{2}\right) \wedge D C\left(f^{2}\right)$ is the inclusion of a summand, where $D C\left(f^{2}\right)$ denotes the $p$-local Spanier-Whitehead dual of $C\left(f^{2}\right)$. This is the case if and only if $f^{2} \wedge D C\left(f^{2}\right): \Sigma^{2 k} X \wedge D C\left(f^{2}\right) \rightarrow X \wedge D C\left(f^{2}\right)$ is trivial. But this places us in the situation of Proposition 2.3, and we have the following result.

Proposition 4.2 Suppose that $X$ is a $K(n-1)_{*}$-acyclic p-locally finite ring spectrum with a two-sided unit and that $g$ is a $v_{n}$ self-map. Then there exists a natural number $N$ such that for each $m>0, C\left(g^{m N}\right)$ has the structure of a ring spectrum with two-sided unit extending the ring spectrum structure on $X$.

Now suppose that $p>2$ and that $X$ is a $p$-local ring spectrum with a two-sided unit. Let $\mu: X \wedge X \rightarrow X$ denote the multiplication and let $\tau: X \wedge X \rightarrow X \wedge X$ denote the switch map. Then $\frac{1}{2}(\mu+\mu \tau): X \wedge X \rightarrow X$ gives $X$ the structure of a homotopy commutative ring spectrum. If $X$ is already homotopy commutative and $C(f)$ has the structure of a ring spectrum with two-sided unit extending that on $X$, 
then this construction provides $C(f)$ with the structure of a homotopy commutative ring spectrum, and this structure again extends the ring spectrum structure on $X$. We will use the construction $\langle\quad\rangle$ of the previous section to obtain the analogue of these observations for $W$-commutative ring spectrum structures, where $W$ is a free contractible $\mathbb{Z} /(2)-C W$ complex.

Let $W$ be as above, and let $Y$ be a CW $S$-module with cellular $\mathbb{Z} /(2)$ action. Let $\zeta$ denote the map

$$
Y=\mathbb{Z} /(2) \wedge_{\mathbb{Z} /(2)} Y \rightarrow W_{+} \wedge_{\mathbb{Z} /(2)} Y
$$

where $\mathbb{Z} /(2) \rightarrow W$ is any choice of equivariant map. We will also need to consider $Y$ with the trivial $\mathbb{Z} /(2)$ action; in this situation, let $\varepsilon$ denote the composition

$$
W_{+} \wedge \mathbb{Z} /(2) Y=B(\mathbb{Z} /(2))_{+} \wedge Y \rightarrow S^{0} \wedge Y=Y,
$$

where $B(\mathbb{Z} /(2))$ is sent to the non-basepoint of $S^{0}$. Let $t: Y \rightarrow Y$ denote the identity map, but with the domain understood to have the given action and the range understood to have the trivial action. As usual, $\sigma: Y \rightarrow Y$ denotes the action by the nontrivial element on $Y$.

Proposition 4.3 (i) $\varepsilon \circ\langle t\rangle \circ \zeta \simeq i d_{Y}+\sigma$

(ii) If $p$ is odd and $Y$ is $p$-local and connective, then $\zeta \circ \frac{1}{2} \varepsilon\langle t\rangle$ is a homotopy equivalence.

Proof (i) This is easy to verify directly.

(ii) Since $Y$ is $p$-local and $p$ is odd,

$$
H_{*}\left(\mathbb{Z} /(2), H_{S}(Y)\right)=\left(H_{S}(Y)\right)_{\mathbb{Z} /(2)}
$$

concentrated in degree 0 . The spectral sequence

$$
H_{*}\left(\mathbb{Z} /(2), H_{*}(Y)\right) \Rightarrow H_{*}\left(W_{+} \wedge_{\mathbb{Z} /(2)} Y\right)
$$

thus collapses and $H_{*}\left(W_{+} \wedge_{\mathbb{Z} /(2)} Y\right)=\left(H_{*}(Y)\right)_{\mathbb{Z} /(2)}$. Under this identification, $\zeta$ induces the quotient map $H_{*} Y \rightarrow\left(H_{*} Y\right)_{\mathbb{Z} /(2)}$, so that $\zeta \circ \frac{1}{2} \varepsilon\langle t\rangle$ induces the identity map on homology and is therefore a homotopy equivalence.

Proposition 4.4 Let $p$ be an odd prime. Suppose that $X$ and $Y$ are p-local ring spectra with two-sided units and that $i: X \rightarrow Y$ is a map of ring spectra. If $X$ is connective and its multiplication $\mu: X \wedge X \rightarrow X$ is homotopy commutative, then there exists a unique $W$-commutative ring spectrum structure extending $\mu$, and there exists a $W$-commutative ring spectrum structure on $Y$ extending that on $X$.

Remark 4.5 Together with Proposition 4.2, this proves the odd primary analogue of Theorem 1.1. 
Proof of Proposition 4.4 Define $\xi_{X}$ to be the composition

$$
W_{+} \wedge \mathbb{Z} /(2)(X \wedge X) \stackrel{\frac{1}{2} \varepsilon\langle t\rangle}{\longrightarrow} X \wedge X \stackrel{\mu}{\longrightarrow} X
$$

Then $\xi_{X} \circ \zeta$ is homotopic to $\frac{1}{2}(\mu+\mu \tau)$ which in turn is homotopic to $\mu$, so $\xi_{X}$ extends $\mu$. This extension is also unique: any two extensions will agree when precomposed with $\zeta$, but, by Proposition 4.3(ii), $\zeta$ is the projection onto a wedge summand.

Now let $v: Y \wedge Y \rightarrow Y$ be the multiplication on $Y$, and let $\xi_{Y}$ be the composition

$$
W_{+} \wedge \mathbb{Z} /(2)(Y \wedge Y) \stackrel{\frac{1}{2} \varepsilon\langle t\rangle}{\longrightarrow} Y \wedge Y \stackrel{v}{\longrightarrow} Y
$$

An easy argument then show that $\xi_{Y}$ extends $\xi_{X}$.

\section{Outline of Proof of Theorem 1.1}

In this section, we begin the main work of this paper. Throughout, we will fix a connected finite free $\mathbb{Z} /(2)-C W$ complex $E$ together with an equivariant map $\iota: E \rightarrow$ $C_{M}(2) . X$ will also be taken to be a $p$-locally finite $K(n-1)_{*}$-acyclic CW $S^{0}$-module with an $E$-commutative ring spectrum structure $\xi_{X}: e(X \wedge X)=E_{+} \wedge \mathbb{Z} /(2) X^{(2)} \rightarrow X$, although these assumptions will not be needed for most of the auxiliary results on cofibrations appearing in this section.

We begin the process of extending $\xi_{X}$ to an $E$-commutative ring spectrum structure on $C(f), f: \Sigma^{k} X \rightarrow X$ an appropriate $v_{n}$ self-map, by constructing a map

$$
\xi_{X, f}: e(C(f) \wedge X \cup X \wedge C(f)) \rightarrow C(f)
$$

extending $\xi_{X}$. The first step towards doing so is an easy observation for spaces, and standard arguments extend it to $S$-modules as well (see "Appendix 3").

Proposition 5.1 Let $f: \Sigma^{k} X \rightarrow X$ and consider the $\mathbb{Z} /(2)$-equivariant map

$$
(f \wedge X, X \wedge f):\left(\Sigma^{k} X \wedge X\right) \vee\left(X \wedge \Sigma^{k} X\right) \rightarrow X \wedge X
$$

which is $f \wedge X$ on the first summand and $X \wedge f$ on the second. The cofiber of this map is naturally equivariantly isomorphic to $C(f) \wedge X \cup X \wedge C(f)$.

Now define $\xi_{X, k}: e\left(\Sigma^{k} X \wedge X \vee X \wedge \Sigma^{k} X\right) \rightarrow \Sigma^{k} X$ to be the composition

$$
\begin{aligned}
e\left(\Sigma^{k} X \wedge X \vee X \wedge \Sigma^{k} X\right) & =E_{+} \wedge\left(\Sigma^{k} X \wedge X\right) \\
& =\Sigma^{k}\left(E_{+} \wedge X \wedge X\right) \rightarrow \Sigma^{k} e(X \wedge X) \stackrel{\Sigma^{k} \xi_{X}}{\longrightarrow} \Sigma^{k} X .
\end{aligned}
$$


Proposition 5.2 Suppose $g: \Sigma^{|g|} X \rightarrow X$ is a $v_{n}$ self-map. Then there exists $N>0$ such that, if $f=g^{m N}$, the diagram

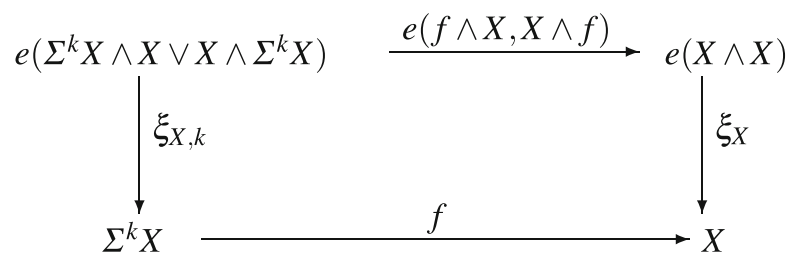

is homotopy commutative. This in turn induces a map $\xi_{X, f}: e(C(f) \wedge X \cup X \wedge$ $C(f)) \rightarrow C(f)$ giving a homotopy commutative diagram

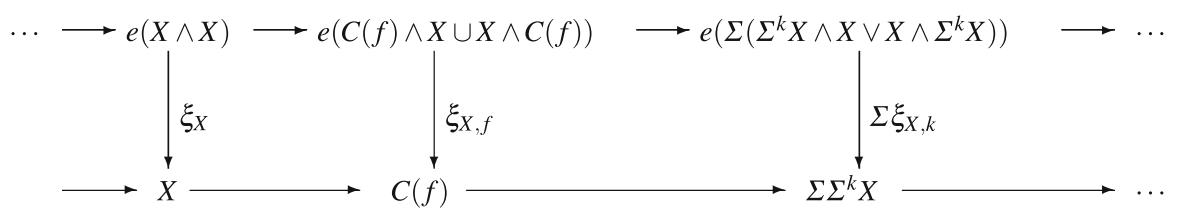

of cofibration sequences.

Proof By nilpotence technology, $N$ may be chosen so that if $f=g^{m N}$, then

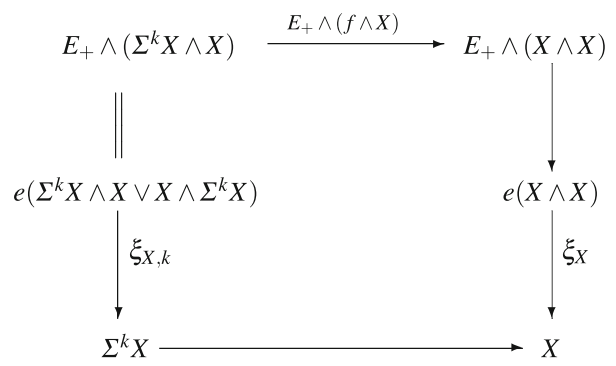

is homotopy commutative. This immediately implies the desired result.

We next consider the $\mathbb{Z} /(2)$-equivariant cofiber sequence

$$
\begin{aligned}
\cdots & \rightarrow C(f) \wedge X \cup X \wedge C(f) \rightarrow C(f) \wedge C(f) \\
& \rightarrow C(f) \wedge C(f) \cup C[C(f) \wedge X \cup X \wedge C(f)] \stackrel{\delta_{f}}{\longrightarrow} \cdots
\end{aligned}
$$

Let $q_{f}$ denote the map

$$
C(f) \wedge C(f) \cup C[C(f) \wedge X \cup X \wedge C(f)] \rightarrow \frac{C(f) \wedge C(f)}{C(f) \wedge X \cup X \wedge C(f)}
$$


followed by the identification

$$
\frac{C(f) \wedge C(f)}{C(f) \wedge X \cup X \wedge C(f)}=\Sigma \Sigma^{k} X \wedge \Sigma \Sigma^{k} X
$$

Proposition $5.3 q_{f}$ is a $\mathbb{Z} /(2)$-equivariant homotopy equivalence.

Proof We first indicate the proof for spaces. That $q_{f}$ is a homotopy equivalence is standard; the only issue is that it is in fact an equivariant homotopy equivalence. But this follows from the equivariant way that the product of two NDR-pairs is expressed as an NDR-pair (see [13, Theorem 6.3]). We then use the techniques of "Appendix 3" to extend the result to the category of $S$-modules.

Since we will be working at the point-set level, it will often be convenient to use the $S$-module $C(f) \wedge C(f) \cup C[C(f) \wedge X \cup X \wedge C(f)]$; by an abuse of notation, we will write

$$
C(f) \wedge C(f) \cup C[C(f) \wedge X \cup X \wedge C(f)] \equiv\left(\Sigma^{k+1} X \wedge \Sigma^{k+1} X\right)^{\prime} .
$$

We will also need to consider maps between cofibration sequences at the point-set level. For example, the map

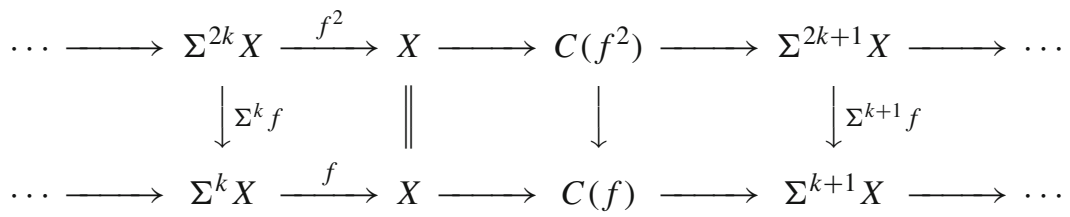

induces a map

$$
\begin{aligned}
& C\left(f^{2}\right) \wedge C\left(f^{2}\right) \cup C\left[C\left(f^{2}\right) \wedge X \cup X \wedge C\left(f^{2}\right)\right] \\
& \rightarrow C(f) \wedge C(f) \cup C[C(f) \wedge X \cup X \wedge C(f)]
\end{aligned}
$$

which makes the diagram

$$
\begin{gathered}
C\left(f^{2}\right) \wedge C\left(f^{2}\right) \cup C\left[C\left(f^{2}\right) \wedge X \cup X \wedge C\left(f^{2}\right)\right] \stackrel{q_{f}^{2}}{\longrightarrow} \Sigma^{2 k+1} X \wedge \Sigma^{2 k+1} X \\
\downarrow \\
\qquad(f) \wedge C(f) \cup C[C(f) \wedge X \cup X \wedge C(f)] \stackrel{\Sigma^{k+1} f \wedge \Sigma^{k+1} f}{\longrightarrow} \Sigma^{k+1} X \wedge \Sigma^{k+1} X
\end{gathered}
$$

commute. We will therefore denote this map by $\left(\Sigma^{k+1} f \wedge \Sigma^{k+1} f\right)^{\prime}$.

We will obtain our extension of $\xi_{X}$ by proving that $\Sigma \xi_{X, f} \circ e\left(\delta_{f}\right): e\left(\Sigma^{k+1} X \wedge\right.$ $\left.\Sigma^{k+1} X\right)^{\prime} \rightarrow \Sigma C(f)$ is null homotopic for $f$ an appropriate $v_{n}$ self-map. The heart of this proof will involve examination of the map $e\left(\delta_{f}\right)$. We begin with an observation whose proof is similar to Propositions 5.1 and 5.3. Recall from the beginning of Sect. 3 our use of the notation $\Sigma_{1}, \Sigma_{2}$ etc. in indicating how suspension coordinates are shuffled. 
Proposition 5.4 The composition

$$
\begin{gathered}
\left(\Sigma^{k+1} X \wedge \Sigma^{k+1} X\right)^{\prime} \stackrel{\delta_{f}}{\longrightarrow} \Sigma(C(f) \wedge X \cup X \wedge C(f)) \stackrel{\Sigma p_{f}}{\longrightarrow} \\
\Sigma^{2}\left(\Sigma^{k} X \wedge X \vee X \wedge \Sigma^{k} X\right) \stackrel{\Sigma^{2} \pi_{1}}{\longrightarrow} \Sigma^{2}\left(\Sigma^{k} X \wedge X\right)
\end{gathered}
$$

is naturally homotopic to the composition

$$
\begin{aligned}
& \left(\Sigma^{k+1} X \wedge \Sigma^{k+1} X\right)^{\prime} \stackrel{q_{f}}{\longrightarrow}\left(\Sigma^{k+1} X \wedge \Sigma^{k+1} X\right) \stackrel{\Sigma^{k+1} X \wedge \Sigma f}{\longrightarrow} \\
& \quad \Sigma_{1} \Sigma^{k} X \wedge \Sigma_{2} X=\Sigma_{1} \Sigma_{2}\left(\Sigma^{k} X \wedge X\right),
\end{aligned}
$$

where $p_{f}$ denotes the boundary map in the cofibration sequence of Proposition 5.1 and, as usual, $\pi_{1}$ denotes the projection onto the first summand.

Proposition 5.5 The composition

$$
\begin{aligned}
& e\left(\Sigma^{k+1} X \wedge \Sigma^{k+1} X\right)^{\prime} \stackrel{e\left(\delta_{f}\right)}{\longrightarrow} e(\Sigma[C(f) \wedge X \cup X \wedge C(f)]) \stackrel{e\left(\Sigma p_{f}\right)}{\longrightarrow} \\
& \quad e\left(\Sigma^{2}\left(\Sigma^{k} X \wedge X \vee X \wedge \Sigma^{k} X\right)\right)
\end{aligned}
$$

is naturally homotopic to the composition

$$
\begin{aligned}
& e\left(\Sigma^{k+1} X \wedge \Sigma^{k+1} X\right)^{\prime} \stackrel{e\left(q_{f}\right)}{\longrightarrow} e\left(\Sigma_{1} \Sigma^{k} X \wedge \Sigma_{2} \Sigma^{k} X\right) \stackrel{\left\langle\Sigma_{1} \Sigma^{k} X \wedge \Sigma_{2} f\right\rangle}{\longrightarrow} \\
& \quad e\left[\Sigma_{1} \Sigma_{2}\left(\Sigma^{k} X \wedge X \vee X \wedge \Sigma^{k} X\right)\right] .
\end{aligned}
$$

Remark 5.6 By $\left\langle\Sigma_{1} \Sigma^{k} X \wedge \Sigma_{2} f\right\rangle$, we mean the application of Construction 3.3 to the composition

$$
\begin{aligned}
& \Sigma^{k+1} X \wedge \Sigma^{k+1} X \stackrel{\Sigma^{k+1} X \wedge \Sigma f}{\longrightarrow} \Sigma_{1} \Sigma^{k} X \wedge \Sigma_{2} X=\Sigma_{1} \Sigma_{2}\left(\Sigma^{k} X \wedge X\right) \\
& \quad \rightarrow \Sigma_{1} \Sigma_{2}\left(\Sigma^{k} X \wedge X \vee X \wedge \Sigma^{k} X\right),
\end{aligned}
$$

where $\mathbb{Z} /(2)$ acts in the usual way on $\Sigma^{k} X \wedge X \vee X \wedge \Sigma^{k} X$ and trivially on $\Sigma^{2} S^{0}$. We have also, of course, left out the suspensions arising from this construction.

Proof of Proposition 5.5 Since $\Sigma p_{f} \circ \delta_{f}$ is defined at an appropriate level of prespectra, Lemma 3.6 is applicable. The desired result then follows from Proposition 5.4.

Proposition 5.7 Suppose that $f=h^{2}$, where $h: \Sigma^{j} X \rightarrow X$ with $j$ even, and that

$$
h \wedge h: \Sigma_{1}^{j} \Sigma_{2}^{j}(X \wedge X)=\Sigma_{1}^{j} X \wedge \Sigma_{2}^{j} X \rightarrow X \wedge X
$$

is homotopic to

$$
X \wedge f: \Sigma^{k}(X \wedge X)=X \wedge \Sigma^{k} X \rightarrow X \wedge X
$$


Then the diagram

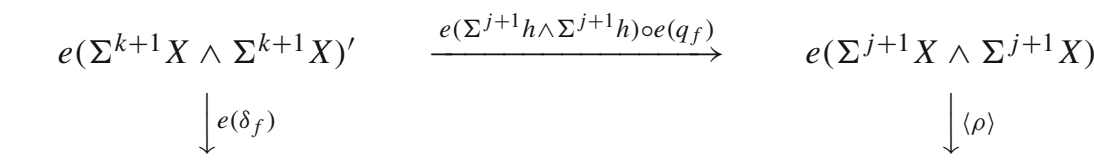

$e(\Sigma[C(f) \wedge X \cup X \wedge C(f)]) \quad \stackrel{e\left(\Sigma p_{f}\right)}{\longrightarrow} \quad e\left(\Sigma^{2}\left(\Sigma^{k} X \wedge X \vee X \wedge \Sigma^{k} X\right)\right)$

is homotopy commutative, where $\rho: \Sigma^{j+1} X \wedge \Sigma^{j+1} X \rightarrow \Sigma^{2}\left(\Sigma^{k} X \wedge X \vee X \wedge \Sigma^{k} X\right)$ is given by the composition

$$
\Sigma_{1} \Sigma_{2}^{j} X \wedge \Sigma_{3} \Sigma_{4}^{j} X \rightarrow \Sigma_{1} \Sigma_{3}\left(\Sigma_{2}^{j} \Sigma_{4}^{j} X \wedge X\right) \rightarrow \Sigma^{2}\left(\Sigma^{k} X \wedge X \vee X \wedge \Sigma^{k} X\right) .
$$

Proof This follows from Proposition 5.5 and the fact that, since $j$ is even, the map $\rho \circ\left(\Sigma^{j+1} h \wedge \Sigma^{j+1} h\right)$ is homotopic to the map of Remark 5.6.

In general, if $h: \Sigma^{j} X \rightarrow X$ and $f=h^{2}$, let $K_{h}$ denote the cofiber of the map $e\left(\Sigma^{j+1} h \wedge \Sigma^{j+1} h\right) \circ e\left(q_{f}\right)$; note that there is a canonical map $\kappa_{h}: K_{f} \rightarrow K_{h}$ yielding a commutative diagram

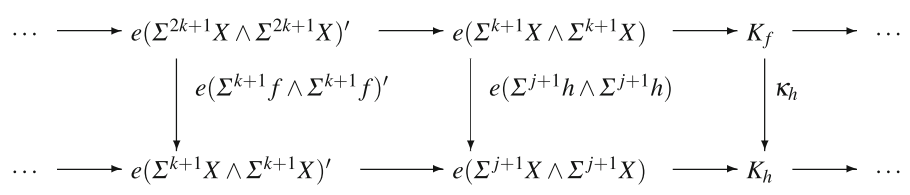

of cofibration sequences.

If $f$ and $h$ satisfy the hypotheses of Proposition 5.7, then there is a homotopy commutative diagram

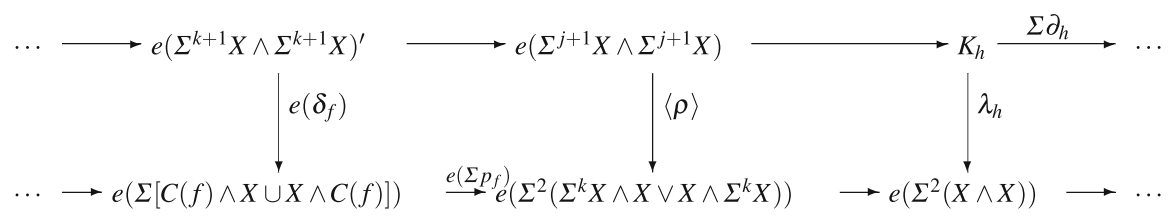

of cofibration sequences. It follows from Proposition 2.3 that, for $h$ an appropriate iterate of a given $v_{n}$ self-map $g, \lambda_{h}$ is unique up to homotopy. In general, of course, given a homotopy commutative square, there may be many maps between the cofibers yielding a homotopy commutative diagram of cofibration sequences. If a homotopy is specified, a map of cofibers may also be specified (see Construction 7.1). Despite the uniqueness of $\lambda_{h}$, we will need to use these considerations, together with a compatibility result for certain homotopies, to prove the next crucial result. This will be carried out in Sects. 7, 8 and 9.

Lemma 5.8 Let $p=2$ and let $g$ be a $v_{n}$ self-map of $X$. (If $n=0$, we assume that $\left.X=\left(\Gamma \Sigma^{\infty} S^{0}\right)_{(2)}\right)$. There exists $N>0$ such that if $h=g^{m N}$, then $\lambda_{h}$ and $\lambda_{f}$ $\left(f=h^{2}\right)$ may be chosen so that $\lambda_{f}=\lambda_{h} \circ \kappa_{h}$. 
In fact, taken together with the next result, this lemma implies that $\lambda_{f} \simeq *$. This is the beginning of our proof that $\Sigma \xi_{X, f} \circ e\left(\delta_{f}\right) \simeq *$ for $f$ an appropriate $v_{n}$ self-map.

Lemma 5.9 Let $g$ be a $v_{n}$ self-map on $X$. There exists $N>0$ such that, if $h=g^{m N}$ : $\Sigma^{j} X \rightarrow X$ and $f=h^{2}: \Sigma^{k} X \rightarrow X$, then the composition

$$
e\left(\Sigma^{j+1} X \wedge \Sigma^{j+1} X\right) \stackrel{\langle\rho\rangle}{\longrightarrow} \Sigma^{2} e\left(\Sigma^{k} X \wedge X \vee X \wedge \Sigma^{k} X\right) \rightarrow \Sigma^{2} e(X \wedge X)
$$

is null homotopic.

Lemma 5.9 will be proved in Sect. 6.

Corollary 5.10 With the hypotheses and notation of Lemma 5.8, there exists $N>0$ such that $\lambda_{f} \simeq *$ and hence $e\left(\delta_{f^{2}}\right) \circ \partial_{f} \simeq *$.

Proof By Lemma 5.9, there is a factorization

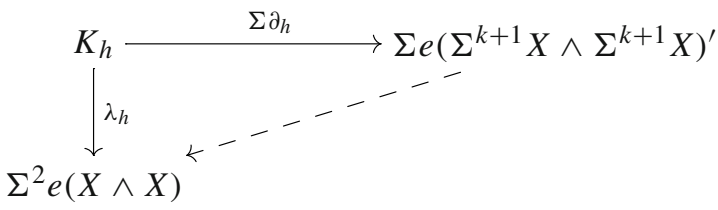

and hence, by Lemma 5.8, we have

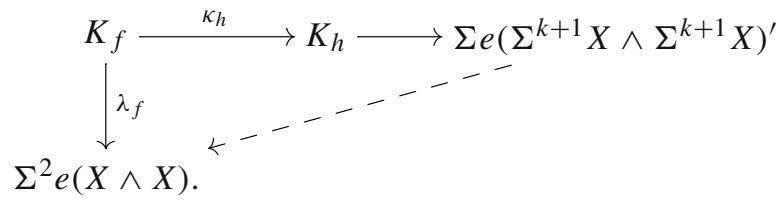

But the composition in the top row is trivial by the very construction of $\kappa_{h}$.

We are now ready to complete the proof of our main result.

Proof of Theorem 1.1 By iterating $g$ if necessary, we may assume by Proposition 3.1 that $\Sigma^{2|g|} e(Y \wedge Y)$ is naturally homotopy equivalent to $e\left(\Sigma^{|g|} Y \wedge \Sigma^{|g|} Y\right)$ for $Y$ a CW $S$-module. Moreover, I claim that

$$
\Sigma^{2|g|} e(\Sigma X \wedge \Sigma X) \simeq e\left(\Sigma^{|g|+1} X \wedge \Sigma^{|g|+1} X\right) \stackrel{e(\Sigma g \wedge \Sigma g)}{\longrightarrow} e(\Sigma X \wedge \Sigma X)
$$

is a $v_{n}$-self-map. If $n>0$, this follows easily by induction up the skeletal filtration of $E$; for $n=0$, the effect of $e(\Sigma g \wedge \Sigma g)$ on rational homotopy is determined by examining its effect on rational cellular chains. We will also write the map in (5.2) as $e(\Sigma g \wedge \Sigma g)$. 
Now choose $N$ large enough, $N$ even, so that if $f=g^{m N}$, then $e\left(\delta_{f}\right) \circ \partial_{h} \simeq *$. This implies that there is a factorization

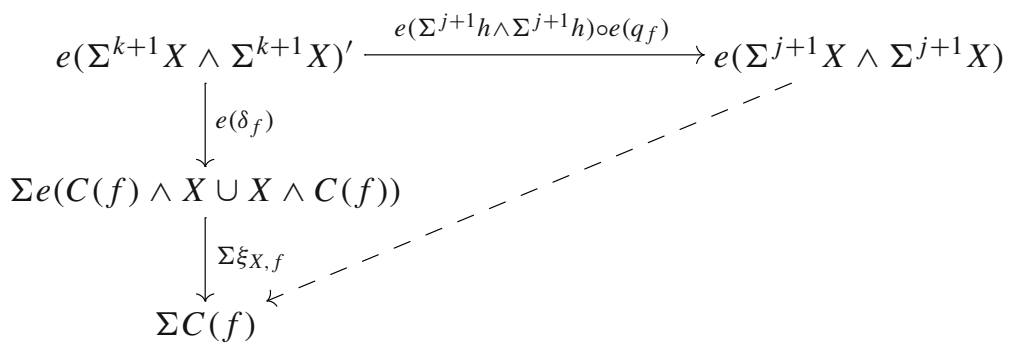

We now identify $e\left(\Sigma^{k+1} X \wedge \Sigma^{k+1} X\right)$ and $e\left(\Sigma^{j+1} X \wedge \Sigma^{j+1} X\right)$ with $\Sigma^{2 k} e(\Sigma X \wedge$ $\Sigma X)$ and $\Sigma^{2 j} e(\Sigma X \wedge \Sigma X)$ in the stable category by iterating the homotopy equivalence $\Sigma^{2|g|} e(Y \wedge Y) \simeq e\left(\Sigma^{|g|} Y \wedge \Sigma^{|g|} Y\right)$. With this identification, $e\left(\Sigma^{j+1} h \wedge \Sigma^{j+1} h\right)$ is just $\Sigma^{2 j} e(\Sigma g \wedge \Sigma g)^{\frac{m N}{2}}$. But by Proposition 2.3, we may choose $N$ so that, in addition,

$$
\Sigma^{2 j} e(\Sigma X \wedge \Sigma X) \wedge D C(f) \stackrel{e(\Sigma g \wedge \Sigma g)^{\frac{m N}{2}} \wedge D C(f)}{\longrightarrow} e(\Sigma X \wedge \Sigma X) \wedge D C(f)
$$

is trivial. This shows that, for such an $N$, the composition $\Sigma \xi_{X, f} \circ e\left(\delta_{f}\right)$ is trivial. Therefore, $\xi_{X, f}$ extends to a map $\xi_{C(f)}^{0}: e(C(f) \wedge C(f)) \rightarrow C(f)$.

Finally, to obtain the unit condition, observe that there is a homotopy commutative diagram

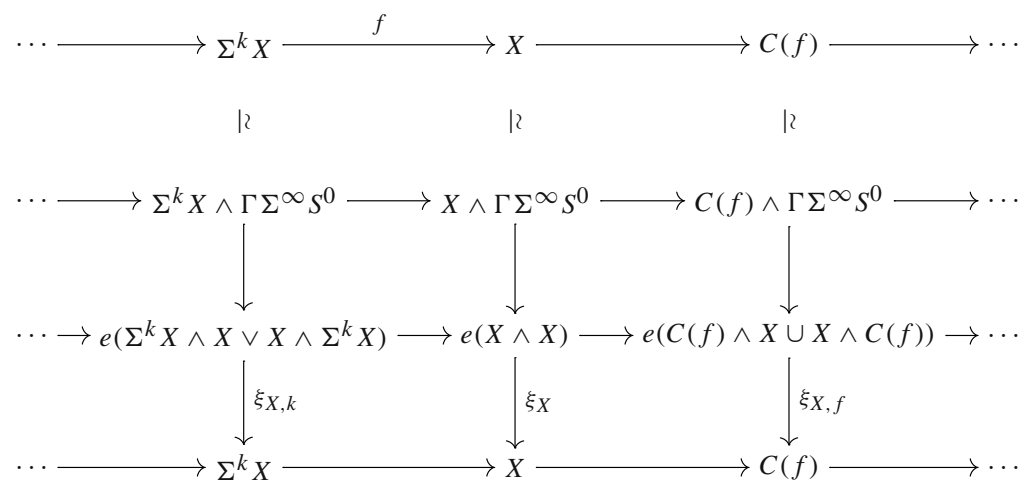

of cofibration sequences, where the two leftmost vertical compositions are homotopic to the identity. It follows that the right vertical composition is a homotopy equivalence 
and that composing $\xi_{C(f)}^{0}$ with the inverse of this equivalence yields the desired $E$ commutative structure $\xi_{C(f)}: e(C(f) \wedge C(f)) \rightarrow C(f)$.

\section{Proof of Lemma 5.9}

We begin the proof by observing that it suffices to show that the composition

$$
\begin{aligned}
& \chi_{0}: e\left(\Sigma^{j+1} X \wedge \Sigma^{j+1} X\right) \stackrel{\langle\rho\rangle}{\longrightarrow} \Sigma^{2} e\left(\Sigma^{k} X \wedge X \vee X \wedge \Sigma^{k} X\right) \\
& \quad=\Sigma^{2} E_{+} \wedge\left(\Sigma^{k} X \wedge X\right) \rightarrow \Sigma^{2+k} e(X \wedge X)
\end{aligned}
$$

is null homotopic. Indeed, $N$ may be chosen so that $\Sigma^{2+k} e(X \wedge X) \simeq \Sigma^{2} e\left(\Sigma^{j} X \wedge\right.$ $\left.\Sigma^{j} X\right)$ (Proposition 3.1) and so that

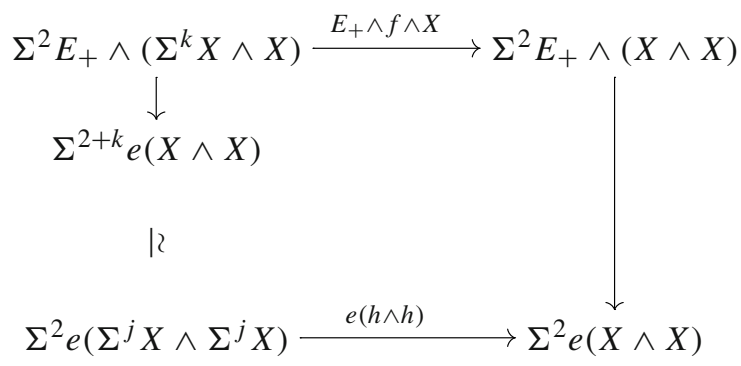

is homotopy commutative (nilpotence technology).

Now let $\alpha_{j}: \Sigma^{j+1} S^{0} \wedge \Sigma^{j+1} S^{0} \rightarrow \Sigma^{j+1} \Sigma_{-}^{j+1} S^{0}$ be a $\mathbb{Z} /(2)$-equivariant homeomorphism-see discussion at the beginning of Sect. 3-and define $\alpha_{j}^{\prime}$ : $\Sigma^{k+2} S^{0} \rightarrow \Sigma^{k+2} S^{0}$ so that the diagram
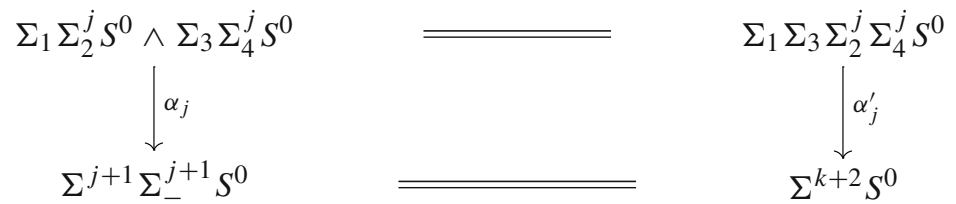

commutes.

Recall that we have fixed a map $\iota: E \rightarrow C_{M}(2)$ and that $\chi_{0}$ is really a map from $\Sigma_{R}^{M} e\left(\Sigma^{j+1} X \wedge \Sigma^{k+1} X\right)$ to $\Sigma_{R}^{M} \Sigma^{2+k} e(X \wedge X)$. There is then a commutative diagram 


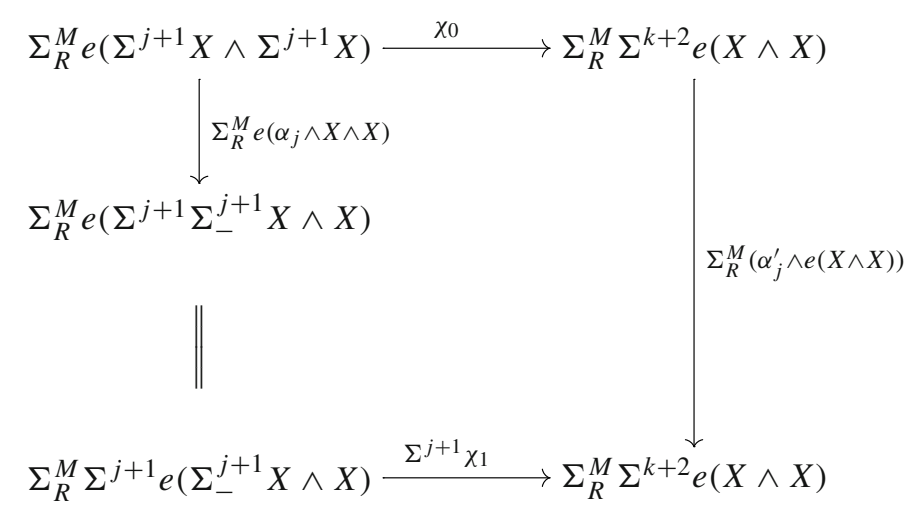

where

$$
\chi_{1}: \Sigma_{R}^{M} e\left(\Sigma_{-}^{j+1} X \wedge X\right) \rightarrow \Sigma_{R}^{M} \Sigma^{j+1} e(X \wedge X)
$$

is given by

$$
\chi_{1}\left[z, s, u, x, t_{1}, \ldots, t_{M}\right]= \begin{cases}{\left[s, u, z, x, \iota_{1}(z)\left(t_{1}, \ldots, t_{M}\right)\right]} & \left(t_{1}, \ldots, t_{M}\right) \in \iota_{1}(z) \\ {\left[-s,-u, z, x, \iota_{2}(z)\left(t_{1}, \ldots, t_{M}\right)\right]} & \left(t_{1}, \ldots, t_{M}\right) \in \iota_{2}(z) .\end{cases}
$$

Here $\left[t_{1}, \ldots, t_{M}\right] \in \Sigma_{R}^{M} S^{0}, z \in E, x \in X \wedge X,[s, u]=\left[s, u_{1}, \ldots, u_{j}\right] \in \Sigma^{j+1} S^{0}$, and $-u=\left(-u_{1}, \ldots,-u_{j}\right)$.

The map $\chi_{1}$ is homotopic to the map $\chi_{2}$ given by

$$
\chi_{2}\left[z, s, u, x, t_{1}, \ldots, t_{M}\right]= \begin{cases}{\left[2 s+1, u, z, x, \iota_{1}(z)\left(t_{1}, \ldots, t_{M}\right)\right]} & -1 \leq s \leq 0 \\ {\left[1-2 s,-u, z, x, \iota_{2}(z)\left(t_{1}, \ldots, t_{M}\right)\right]} & 0 \leq s \leq 1\end{cases}
$$

if

$$
L(t, s)= \begin{cases}(1-t) s+t(2 s+1) & (1-t) s+t(2 s+1) \leq 1 \\ 1 & (1-t) s+t(2 s+1) \geq 1\end{cases}
$$

for $t \in I, s \in[-1,1]$, then the mapping

$$
\begin{aligned}
& \left(\left[z, s, u, x, t_{1}, \ldots, t_{M}\right], t\right) \mapsto \\
& \begin{cases}{\left[L(t, s), u, z, x, \iota_{1}(z)\left(t_{1}, \ldots, t_{M}\right)\right]} & \left(t_{1}, \ldots, t_{M}\right) \in \iota_{1}(z) \\
{\left[L(t,-s),-u, z, x, \iota_{2}(z)\left(t_{1}, \ldots, t_{M}\right)\right]} & \left(t_{1}, \ldots, t_{M}\right) \in \iota_{2}(z)\end{cases}
\end{aligned}
$$

defines a homotopy from $\chi_{1}$ to $\chi_{2}$. 
The map $\chi_{2}$ is in turn homotopic to the map $\Sigma_{R}^{M} \chi$, where $\chi: e\left(\Sigma_{-}^{j+1} X \wedge X\right) \rightarrow$ $\Sigma^{j+1} e(X \wedge X)$ is defined by

$$
\chi[z, s, u, x]= \begin{cases}{[2 s+1, u, z, x]} & -1 \leq s \leq 0 \\ {[1-2 s,-u, z, x]} & 0 \leq s \leq 1\end{cases}
$$

To see this, just make use of linear homotopies from $\iota_{1}(z): \Sigma_{R}^{M} S^{0} \rightarrow \Sigma_{R}^{M} S^{0}$ and $\iota_{2}(z): \Sigma_{R}^{M} S^{0} \rightarrow \Sigma_{R}^{M} S^{0}$ to the identity. We will show that, for appropriate $N$-and hence $j-\chi$ is null homotopic.

Let $\theta: E \rightarrow S^{l}$ be equivariant and, choosing $N$ larger if necessary, assume that $a_{l+1} \mid j$. (As before, $a_{l+1}$ is the order of the canonical line bundle in $\widetilde{K O}\left(\mathbb{R} P^{l+1}\right)$ ). Let $G: S^{l} \rightarrow S O(j+J)$ be as in Lemma 3.2; i.e., $G$ is homotopic to the constant map at the identity and

$$
G(z)(u, v)=G(-z)(-u, v)
$$

for all $z \in S^{l}, u \in \mathbb{R}^{j}$, and $v \in \mathbb{R}^{J}$. As in the proof of Proposition 3.1, $S O(j+J)$ acts on $S\left(\mathbb{R}^{j+J}\right) \equiv \Sigma^{j+J} S^{0}$; we may then regard $G$ as mapping into Map $\left(\Sigma^{j+J} S^{0}, \Sigma^{j+J} S^{0}\right)$. $G$ is still homotopic to the constant map at the identity and Eq. 6.1 holds with $u \in \Sigma^{j} S^{0}$, $v \in \Sigma^{J} S^{0}$.

Let

$$
H: S^{l} \times\left[0, \frac{1}{2}\right] \rightarrow \operatorname{Map}\left(\Sigma^{j+J} S^{0}, \Sigma^{j+J} S^{0}\right)
$$

be a homotopy with $H(z, 0)=i d_{\Sigma^{j+J}} S^{0}$ and $H\left(z, \frac{1}{2}\right)=G(z)$, and define

$$
\widetilde{H}: \Sigma^{J} e\left(\Sigma_{-}^{j+1} X \wedge X\right) \wedge I_{+} \rightarrow \Sigma_{1}^{j+1} \Sigma_{2}^{J} e(X \wedge X)=\Sigma_{2}^{J} \Sigma_{1}^{j+1} e(X \wedge X)
$$

as follows. If $0 \leq t \leq \frac{1}{2}$

$$
\widetilde{H}[v, z, s, u, x, t]= \begin{cases}{[2 s+1, H(\theta(z), t)(u, v), z, x]} & -1 \leq s \leq 0 \\ {[1-2 s, H(-\theta(z), t)(-u, v), z, x]} & 0 \leq s \leq 1,\end{cases}
$$

where $v \in \Sigma^{J} S^{0}, z \in E, s \in \Sigma S^{0}, u \in \Sigma^{j} S^{0}$, and $x \in X \wedge X$. If $\frac{1}{2} \leq t \leq 1$, $\widetilde{H}[v, z, s, u, x, t]= \begin{cases}{[(4-4 t) s+3-4 t, G(\theta(z))(u, v), z, x]} & -1 \leq s \leq 0 \\ {[(4 t-4) s+3-4 t, G(-\theta(z))(-u, v), z, x]} & 0 \leq s \leq 1\end{cases}$

$\widetilde{H}$ is a homotopy from $\Sigma^{J} \chi$ to the trivial map, completing the proof. 


\section{Homotopies and cofiber sequences}

In this section, we set up our conventions for using homotopies to define maps between cofibers. We then observe that compatible homotopies lead to homotopic maps between cofibers. This will be used in Sect. 9 to prove Lemma 5.8. Since $\mathbb{Z} /(2)$ actions play no role in these general considerations, we will, up through Proposition 7.2, give $\Sigma$ coordinates in $[0,1]$, and, when dealing with cones, take 0 to be the cone point.

Construction 7.1 Suppose that

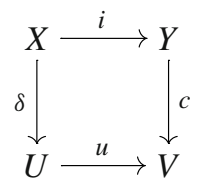

is a homotopy commutative diagram of $S$-modules, and let $H$ be a homotopy from $u \delta$ to ci. Define

$$
\bar{c}_{H}: Y \cup_{i} C X \rightarrow V \cup_{u} C U
$$

by

$$
\begin{aligned}
\bar{c}_{H}(y) & =c(y) \\
\bar{c}_{H}([x, s]) & =\left\{\begin{array}{lll}
{[\delta(x), 2 s]} & y \in X, & 0 \leq s \leq \frac{1}{2} \\
H(x, 2 s-1) & x \in X, & \frac{1}{2} \leq s \leq 1 .
\end{array}\right.
\end{aligned}
$$

The diagram

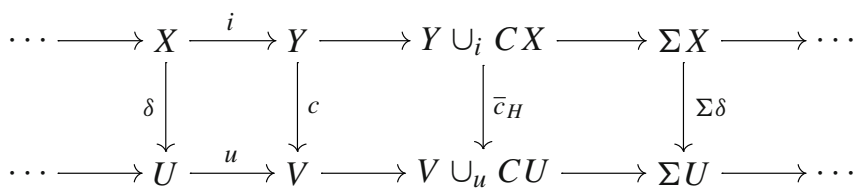

is homotopy commutative, and $\bar{c}_{H}$ will be called the map induced by $H$.

If diagram (7.1) is explicitly assumed to commute on the nose, then define $\bar{c}$ : $Y \cup_{i} C X \rightarrow V \cup_{u} C U$ by $\bar{c}(y)=c(y)$ for $y \in Y$ and $c([x, s])=[\delta(x), s]$ for $x \in X$ and $0 \leq s \leq 1$. Since $\bar{c}$ is homotopic to the map induced by the stationary homotopy, this should not lead to any confusion.

More generally, if $H^{\prime}$ is another homotopy from $u \delta$ to $c i$ with $H \simeq \simeq_{p} H^{\prime}$ in $\operatorname{Map}^{S}(X, V)$, then $\bar{c}_{H} \simeq \bar{c}_{H^{\prime}}$. We may also replace $\delta$ and $c$ by homotopic maps. More precisely: if $G$ is a homotopy from $\delta$ to $\delta^{\prime}$ and $H^{\prime}$ is a homotopy from $u \delta^{\prime}$ to $c i$ with $H \simeq p(u \circ G) * H^{\prime}$, then $\bar{c}_{H} \simeq \bar{c}_{H^{\prime}}$. Similarly, if $G$ is a homotopy from $c^{\prime}$ to $c$ and $H^{\prime}$ is a homotopy from $u \delta$ to $c^{\prime} i$ with $H \simeq{ }_{p} H^{\prime} *(G \circ i)$, then $\bar{c}_{H} \simeq{\overline{c^{\prime}}}_{H^{\prime}}$. 
Finally, if

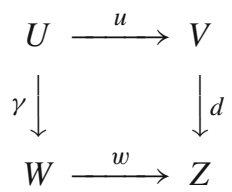

is another homotopy commutative diagram of $S$-modules with homotopy $G$ from $w \gamma$ to $d u$, then $\bar{d}_{G} \circ \bar{c}_{H}: Y \cup_{i} C X \rightarrow Z \cup_{w} C W$ is homotopic to $\overline{(d \circ c)}_{L}$, where $L$ is the homotopy from $w(\gamma \delta)$ to $(d c) i$ given by $L=(G \circ \delta) *(d \circ H)$.

An easy consequence of these observations is the following result, which will be used later.

Proposition 7.2 Consider the diagrams

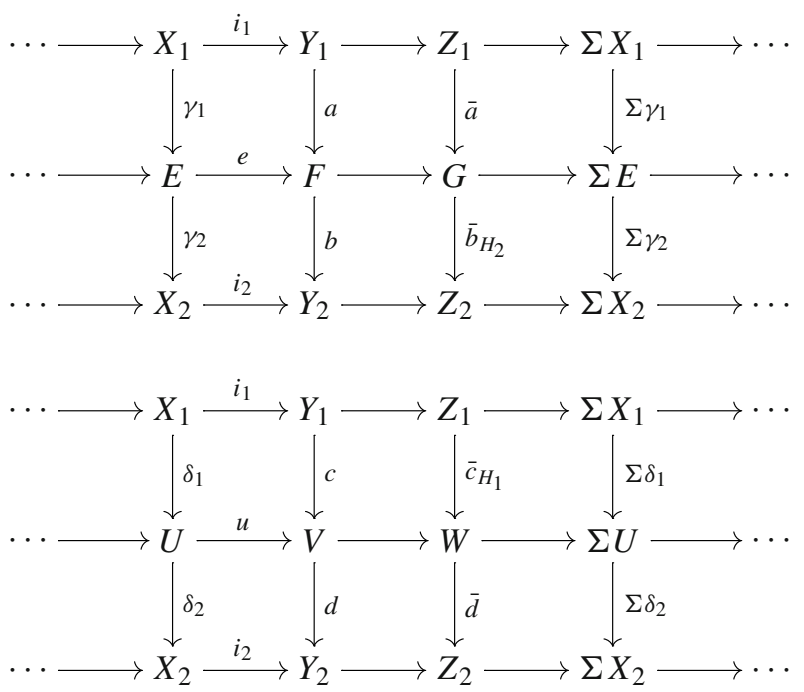

of $S$-modules, where the rows are cofibration sequences, $a i_{1}=e \gamma_{1}, d u=i_{2} \delta_{2}$, $\gamma_{2} \gamma_{1}=\delta_{2} \delta_{1} \equiv \delta, H_{1}$ is a homotopy from $u \delta_{1}$ to $c i_{1}$, and $H_{2}$ is a homotopy from $i_{2} \gamma_{2}$ to be. Suppose that $K$ is a homotopy from dc to ba and consider the map $X_{1} \wedge \dot{\Delta}_{+}^{2} \rightarrow Y_{2}$ defined as follows:

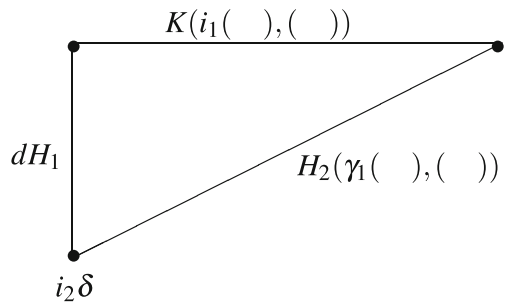

If this map extends to a map $X_{1} \wedge \Delta_{+}^{2} \rightarrow Y_{2}$, then $\bar{d} \circ \bar{c}_{H_{1}}$ is homotopic to $\bar{b}_{H_{2}} \circ \bar{a}$. 
We will apply Proposition 7.2 to the following diagrams:
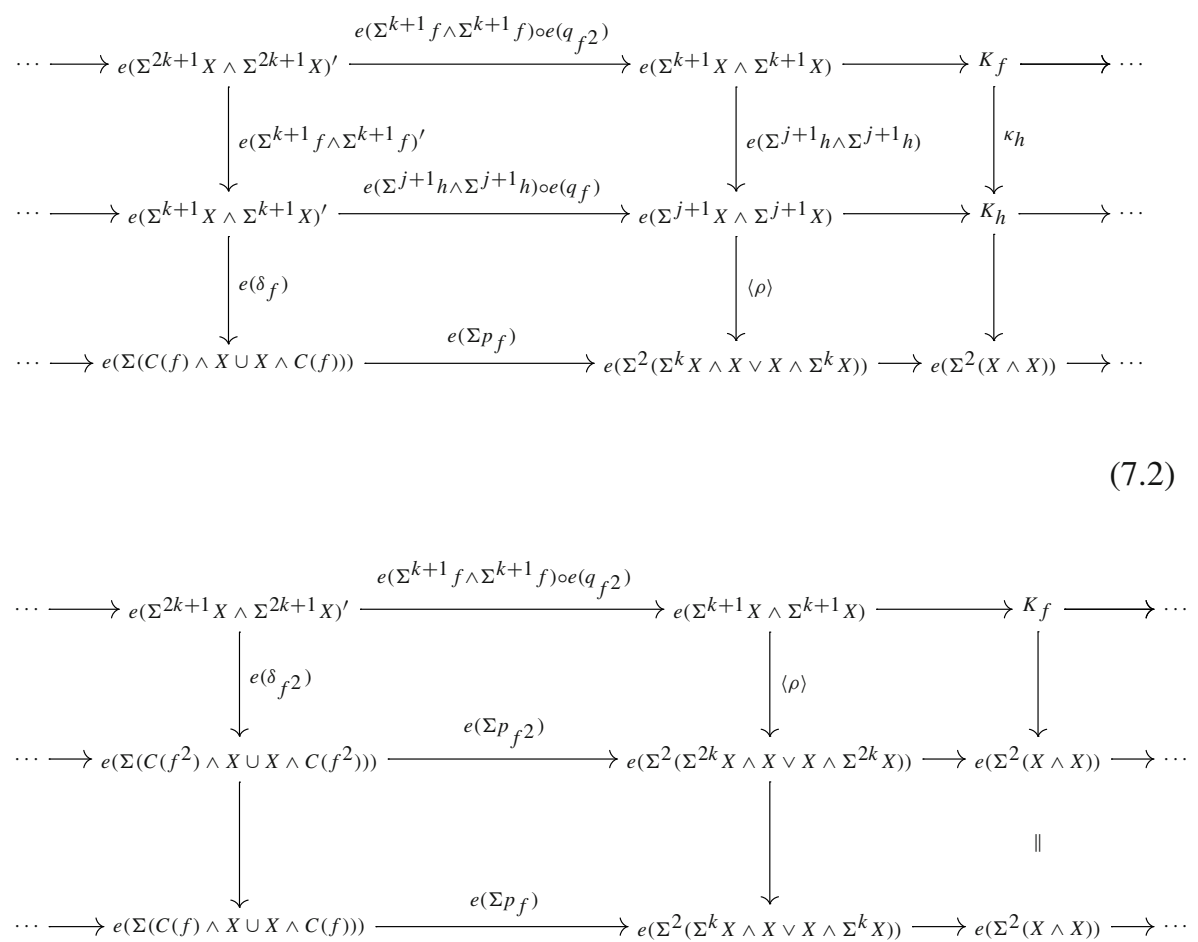

To do this, we will need to examine homotopies making the bottom left square of the first diagram and the top left square of the second diagram commute, together with a homotopy between the middle vertical compositions. We will carry this out in the next two sections.

\section{Homotopies between $v_{n}$ self-maps}

To show that the diagrams (7.2) satisfy the compatibility condition of Proposition 7.2, we will need to examine homotopies between certain $v_{n}$ self-maps. More precisely, suppose that $X$ is a $K(n-1)_{*}$-acyclic finite 2-local CW $S$-module and that $g: \Sigma^{j} X \rightarrow$ $X$ is a $v_{n}$ self-map such that $g \wedge X$ is homotopic to $X \wedge g$. (Once again, if $n=0$, we will assume that $\left.X=\Gamma \Sigma^{\infty} S_{(2)}^{0}\right)$. Let $U: I \rightarrow \operatorname{Map}^{S}\left(\Sigma^{j} X \wedge X, X \wedge X\right)$ be a homotopy from $g \wedge X$ to $X \wedge g$. Using the notation of Sect. 2, our goal will be to show that there exists $N>0$ such that

$$
\begin{aligned}
& \left(g^{N} \wedge X\right) \circ \Sigma^{N j} U^{(N)} \simeq{ }_{p} U^{(N)} \circ \Sigma^{N j}\left(g^{N} \wedge X\right) \\
& \left(X \wedge g^{N}\right) \circ \Sigma^{N j} U^{(N)} \simeq{ }_{p} U^{(N)} \circ \Sigma^{N j}\left(X \wedge g^{N}\right)
\end{aligned}
$$

as paths in $\operatorname{Map}^{S}\left(\Sigma^{2 N j} X \wedge X, X \wedge X\right)$. 
At this point, the alert reader may have noticed that the above two relations do not make sense as written. For example,

$$
\left(g^{N} \wedge X\right) \circ \Sigma^{N j} U^{(N)}(1)=\left(g^{N} \wedge X\right) \circ \Sigma^{N j}\left(X \wedge g^{N}\right)
$$

and

$$
U^{(N)}(1) \circ \Sigma^{N j}\left(g^{N} \wedge X\right)=\left(X \wedge g^{N}\right) \circ \Sigma^{N j}\left(g^{N} \wedge X\right) .
$$

But $\left(g^{N} \wedge X\right) \circ \Sigma^{N j}\left(X \wedge g^{N}\right)$ is the composition

$$
\Sigma_{1}^{N j} \Sigma_{2}^{N j} X \wedge X=\Sigma_{1}^{N j} X \wedge \Sigma_{2}^{N j} X \stackrel{g^{N} \wedge g^{N}}{\longrightarrow} X \wedge X
$$

whereas $\left(X \wedge g^{N}\right) \circ \Sigma^{N j}\left(g^{N} \wedge X\right)$ is the composition

$$
\Sigma_{1}^{N j} \Sigma_{2}^{N j} X \wedge X=\Sigma_{2}^{N j} X \wedge \Sigma_{1}^{N j} X \stackrel{g^{N} \wedge g^{N}}{\longrightarrow} X \wedge X
$$

If $j>0$, these compositions are not equal: they differ by a permutation of suspension coordinates. Of course, if $j$ is even, any permutation of the factors of $\left(S^{j}\right)^{(l)}$, the $l$-fold smash product of $S^{j}$, is homotopic to the identity. The necessary modification of (8.1) is a consequence of the following result.

Proposition 8.1 With notation as above, there exists $N>0$ such that, for any $m$,

$$
\begin{aligned}
& U^{(m N)} \circ \Sigma^{N m j} \bar{U}^{(m N)} \circ(T \wedge X \wedge X) \\
& \quad \in \pi_{1}\left(\operatorname{Map}_{g^{m N} \wedge g^{m N}}^{S}\left(S^{N m j} \wedge S^{N m j} \wedge X \wedge X, X \wedge X\right)\right)
\end{aligned}
$$

is trivial, where $T$ is some homotopy from the identity to the switch map

$$
\tau: S_{1}^{N m j} \wedge S_{2}^{N m j} \rightarrow S_{2}^{N m j} \wedge S_{1}^{N m j}
$$

Remark 8.2 If $u: \Sigma^{k} X \rightarrow W$ and $v: \Sigma^{l} Y \rightarrow Z$, then by $u \wedge v: \Sigma^{k+l} X \wedge Y \rightarrow W \wedge Z$, we mean the composition

$$
\Sigma^{k+l} X \wedge Y=\Sigma_{1}^{k} \Sigma_{2}^{l} X \wedge Y=\Sigma_{1}^{k} X \wedge \Sigma_{2}^{l} Y \stackrel{u \wedge v}{\longrightarrow} W \wedge Z .
$$

Before proving this result, we give the following consequence, which is what we will use in the next section.

Corollary 8.3 With notation as above,

$$
\left(g^{m N} \wedge X\right) \circ \Sigma^{N m j} U^{(m N)} \simeq_{p} U^{(m N)} \circ\left(\Sigma^{N m j} g^{m N} \wedge X\right) \circ(T \wedge X \wedge X)
$$

and

$$
\left(X \wedge g^{m N}\right) \circ \Sigma^{N m j} U^{(m N)} \circ(\tau \wedge X \wedge X) \simeq{ }_{p} U^{(m N)} \circ\left(\Sigma^{N m j} X \wedge g^{m N}\right) \circ(T \wedge X \wedge X)
$$


Proof of Corollary To prove the first statement, observe that

$$
\begin{aligned}
U^{(m N)} \circ \Sigma^{N m j} \bar{U}^{(m N)} \circ(T \wedge X \wedge X) \simeq & p\left[\left(g^{m N} \wedge X\right) \circ \Sigma^{N m j} \bar{U}^{(m N)}\right] \\
& *\left[U^{(m N)} \circ\left(\Sigma^{N m j} g^{m N} \wedge X\right) \circ(T \wedge X \wedge X)\right] .
\end{aligned}
$$

To prove the second statement observe that

$$
\begin{aligned}
U^{(m N)} \circ \Sigma^{N m j} \bar{U}^{(m N)} \circ(T \wedge X \wedge X) \simeq p & {\left[U^{(m N)} \circ\left(\Sigma^{N m j} X \wedge g^{m N}\right) \circ(T \wedge X \wedge X)\right] } \\
& *\left[\left(X \wedge g^{m N}\right) \circ \Sigma^{N m j} \bar{U}^{(m N)} \circ(T(1) \wedge X \wedge X)\right] .
\end{aligned}
$$

We now turn to the proof of Proposition 8.1. The main ingredients are Proposition 2.5 as applied to $v_{n}$ self-maps together with a little bit of understanding of homotopies between permutations of the factors of $\left(S^{j}\right)^{(l)}$. These permutations add a lot of clutter to the proofs of Proposition 8.1 and Lemma 5.8 in Sect. 9; the reader may therefore find them easier to follow and the ideas involved more transparent if all considerations involving permutations are ignored on the first pass through. With this warning, we begin our work.

As before, let $\Sigma_{l}$ denote the symmetric group on $l$ letters. If $\sigma \in \Sigma_{l}$, we will also write $\sigma$ for the map $\left(S^{j}\right)^{(l)} \rightarrow\left(S^{j}\right)^{(l)}$ sending $S_{1}^{j} \wedge \cdots \wedge S_{l}^{j}$ to $S_{\sigma^{-1}(1)}^{j} \wedge \cdots \wedge S_{\sigma^{-1}(l)}^{j}$. If $\sigma^{2}=i d, \sigma$ will be called a conjugation. This is equivalent to saying that $\sigma$ is the composition of commuting transpositions.

Lemma 8.4 Let $\sigma \in \Sigma_{l}$, and let $T^{\sigma}: I \rightarrow \operatorname{Map}\left(S^{j l}, S^{j l}\right)$ be any homotopy from id to $\sigma$. Then $T^{\sigma} \circ \overline{T^{\sigma}} \in \pi_{1}\left(\operatorname{Map}_{\sigma}\left(S^{j l}, S^{j l}\right)\right)$ is trivial. If $\sigma$ is a conjugation and $4 \mid j$, then $T^{\sigma} \circ T^{\sigma} \in \pi_{1}\left(\operatorname{Map}_{i d}\left(S^{j l}, S^{j l}\right)\right)$ is also trivial.

Proof Assume that $j>0$; otherwise everything is trivial (remember, we are working in the category of pointed spaces here).

To prove the first assertion, begin by observing that

$$
T^{\sigma} *\left(\sigma \circ T^{\sigma}\right) \simeq_{p} T^{\sigma} \circ T^{\sigma} \simeq_{p} T^{\sigma} *\left(T^{\sigma} \circ \sigma\right)
$$

and therefore that $\sigma \circ T^{\sigma} \simeq_{p} T^{\sigma} \circ \sigma$. But

$$
T^{\sigma} \circ \overline{T^{\sigma}} \simeq p\left(T^{\sigma} \circ \sigma\right) *\left(\sigma \circ \overline{T^{\sigma}}\right)
$$

hence

$$
T^{\sigma} \circ \overline{T^{\sigma}} \simeq_{p}\left(\sigma \circ T^{\sigma}\right) *\left(\sigma \circ \overline{T^{\sigma}}\right) \simeq_{p} \sigma \circ\left(T^{\sigma} * \overline{T^{\sigma}}\right) \simeq_{p} c_{\sigma}
$$

For the second assertion, we first construct a homotopy $T_{0}^{\tau}$ with the property that $T_{0}^{\tau} \circ T_{0}^{\tau} \in \pi_{1}\left(\operatorname{Map}_{i d}\left(S^{2 j}, S^{2 j}\right)\right)$ is trivial. Here $\tau$ is the switch map and $l=2$.

Let $\gamma_{2}$ denote the canonical line bundle over $\mathbb{R} P^{2}$. Since $4 \gamma_{2}=0$ in $\widetilde{K O}\left(\mathbb{R} P^{2}\right)$ and $B O(4) \rightarrow B O$ is a 4-equivalence, we have that $4 \gamma_{2} \approx \varepsilon^{4}$. By Lemma 3.2, there 
exists $G_{0}: S^{2} \rightarrow S O(2 j)$ such that $G_{0}(z)(u, v)=G_{0}(-z)(-u, v)$ for all $u, v \in \mathbb{R}^{j}$. If we choose a point $e \in S^{2}$, we may also assume that $G_{0}(e)=I$. Now $\mathbb{R}^{j} \oplus \mathbb{R}^{j}$ with the $\mathbb{Z} /(2)$-action given by the switch map is equivariantly isometric to $\mathbb{R}_{-}^{j} \oplus \mathbb{R}^{j}$ (see discussion preceding Lemma 3.2); we thus obtain a map $G: S^{2} \rightarrow S O(2 j)$ such that $G(e)=I$ and $G(z)(u, v)=G(-z)(v, u)$ for all $u, v \in \mathbb{R}^{j}$. By regarding $S^{j}$ as the one point compactification of $\mathbb{R}^{j}$, we may regard $G$ as a map from $S^{2}$ to the space $\operatorname{Map}\left(S^{j} \wedge S^{j}, S^{j} \wedge S^{j}\right)$.

Choose a path $\varphi$ in $S^{2}$ from $e$ to $-e$ and define $T_{0}^{\tau}=G \circ \varphi$. Observe that

$$
\left(T_{0}^{\tau} \circ \tau\right)(t)(u, v)=\left(T_{0}^{\tau}\right)(t)(v, u)=G(\varphi(t))(v, u)=G(-\varphi(t))(u, v) .
$$

But $-\varphi(t)$ is a path from $-e$ to $e$ in $S^{2}$ and is therefore path homotopic to the reverse of $\varphi$. Since

$$
T_{0}^{\tau} \circ T_{0}^{\tau} \simeq p T_{0}^{\tau} *\left(T_{0}^{\tau} \circ \tau\right)
$$

it follows that $T_{0}^{\tau} \circ T_{0}^{\tau} \simeq c_{i d}$.

In general, if $\sigma \in \Sigma_{l}$ is a conjugation, then we may compose the above homotopies in an evident way to obtain a homotopy $T_{0}^{\sigma}$ with $\left(T_{0}^{\sigma}\right)^{(2)}$ trivial in $\pi_{1}\left(\operatorname{Map}_{i d}\left(S^{j l}, S^{j l}\right)\right)$. However, we must show that $\left(T^{\sigma}\right)^{(2)}$ is trivial for any homotopy.

To prove this, write $T^{\sigma} \simeq{ }_{p} \omega * T_{0}^{\sigma}$ with $\omega \in \pi_{1}\left(\operatorname{Map}_{i d}\left(S^{j l}, S^{j l}\right)\right)$. Then

$$
\begin{aligned}
\left(T^{\sigma}\right)^{(2)}=\left(\omega * T_{0}^{\sigma}\right)^{(2)} & \simeq{ }_{p}\left(\omega * T_{0}^{\sigma}\right) *\left(\sigma \circ\left(\omega * T_{0}^{\sigma}\right)\right) \\
& \simeq{ }_{p} \omega * T_{0}^{\sigma} *(\sigma \circ \omega) *\left(\sigma \circ T_{0}^{\sigma}\right) \\
& \simeq{ }_{p} \omega *\left[T_{0}^{\sigma} *(\sigma \circ \omega) * \overline{T_{0}^{\sigma}}\right] *\left(T_{0}^{\sigma} *\left(\sigma \circ T_{0}^{\sigma}\right)\right) .
\end{aligned}
$$

But since $\pi_{1}\left(\operatorname{Map}_{i d}\left(S^{j l}, S^{j l}\right)\right)=\mathbb{Z} /(2), \omega *\left[T_{0}^{\sigma} *(\sigma \circ \omega) * \overline{T_{0}^{\sigma}}\right]$ is path homotopic to the constant loop, and therefore

$$
\left(T^{\sigma}\right)^{(2)} \simeq_{p} T_{0}^{\sigma} *\left(\sigma \circ T_{0}^{\sigma}\right) \simeq_{p}\left(T_{0}^{\sigma}\right)^{(2)} \simeq_{p} c_{i d}
$$

We will also need the following easily proved result.

Lemma 8.5 Suppose that $W, X, Y$, and $Z$ are $C W S$-modules and that $V$ and $V^{\prime}$ are loops in $\operatorname{Map}^{S}(X, Y)$ such that $\underbrace{V * \cdots * V}_{m} \equiv m V \simeq_{p} m V^{\prime}$. If $K$ and $L$ are any paths in $\operatorname{Map}^{S}(Y, Z)$ and $\operatorname{Map}^{S}(W, X)$ respectively such that

$$
K(0) \circ V(0) \circ L(0)=K(1) \circ V(1) \circ L(1),
$$

then $m(K \circ V \circ L) \simeq{ }_{p} m\left(K \circ V^{\prime} \circ L\right)$. 
Proof Observe that

$$
\begin{aligned}
K \circ V \circ L & \simeq p(K(0) \circ V(0) \circ L) *(K(0) \circ V \circ L(1)) *(K \circ V(1) \circ L(1)) \\
& \simeq{ }_{p}[\omega *(K(0) \circ V \circ L(1)) * \bar{\omega}] *[\omega *(K \circ V(1) \circ L(1))],
\end{aligned}
$$

where $\omega=K(0) \circ V(0) \circ L$. Since $\pi_{1}\left(\operatorname{Map}_{q}(W, Z)\right)$ is abelian for any $q$ (see Remark 2.6), we have that

$$
m(K \circ V \circ L) \simeq_{p}[\omega *(K(0) \circ m V \circ L(1)) * \bar{\omega}] * m[\omega *(K \circ V(1) \circ L(1))]
$$

But $V^{\prime}(0)=V(0)=V(1)=V^{\prime}(1)$, so by the same argument,

$$
m\left(K \circ V^{\prime} \circ L\right) \simeq_{p}\left[\omega *\left(K(0) \circ m V^{\prime} \circ L(1)\right) \circ \bar{\omega}\right] * m[\omega *(K \circ V(1) \circ L(1))] .
$$

Since $m V \simeq{ }_{p} m V^{\prime}$, the desired result follows.

Proof of Proposition 8.1 First observe that there exists $R$ such that

$$
2^{R} \pi_{1}\left(\operatorname{Map}_{q}^{S}\left(\Sigma^{N j} X \wedge X, X \wedge X\right)\right)=0
$$

for all $N$ and $q: \Sigma^{N j} X \wedge X \rightarrow X \wedge X$. If $n>0$, this follows from the fact that $i d_{X \wedge X} \in[X \wedge X, X \wedge X]_{0}$ is annihilated by a power of two, and if $n=0$, this follows from the fact that $\operatorname{Map}_{q}^{S}\left(\Sigma^{N j} X \wedge X, X \wedge X\right) \simeq \operatorname{Map}_{q}^{S}\left(\Gamma \Sigma^{\infty} S_{(2)}^{0}, \Gamma \Sigma^{\infty} S_{(2)}^{0}\right)$, so that

$$
\pi_{1}\left(\operatorname{Map}_{q}^{S}\left(\Sigma^{N j} X \wedge X, X \wedge X\right)\right)=\mathbb{Z} /(2)
$$

It thus suffices to prove the following claim:

Suppose that $r \geq 0$, that $g \wedge g$ is in the center of $[X \wedge X, X \wedge X]_{*}$ with $4 \mid j$, and that

$$
2^{r+1}\left(U \circ \Sigma^{j} \bar{U} \circ\left(T_{0} \wedge X \wedge X\right)\right) \simeq{ }_{p} c_{g \wedge g}
$$

for some homotopy $T_{0}: I \rightarrow \operatorname{Map}\left(S^{j} \wedge S^{j}, S^{j} \wedge S^{j}\right)$ from the identity to the switch map. Then for each $m>0$, there exists a homotopy $T: I \rightarrow \operatorname{Map}\left(S^{2 m j} \wedge S^{2 m j}, S^{2 m j} \wedge\right.$ $S^{2 m j}$ ) from the identity to the switch map such that

$$
2^{r}\left(U^{(2 m)} \circ \Sigma^{2 m j} \bar{U}^{(2 m)} \circ(T \wedge X \wedge X)\right) \simeq p c_{g^{2 m} \wedge g^{2 m}} .
$$

We first prove the inductive step with $m=1$. Start with the relation

$$
U \circ \Sigma^{j} \bar{U} \circ\left(T_{0} \wedge X \wedge X\right) \circ \Sigma^{2 j} \bar{U} \circ \Sigma^{3 j} U \circ\left(\Sigma^{2 j} \bar{T}_{0} \wedge X \wedge X\right) \simeq_{p} c_{(g \wedge g)^{2}}
$$

guaranteed by Proposition 2.5. The inductive hypothesis implies that

$$
2^{r}\left(U \circ \Sigma^{j} \bar{U} \circ\left(T_{0} \wedge X \wedge X\right)\right) \simeq_{p} 2^{r}\left(\bar{U} \circ \Sigma^{j} U \circ\left(\bar{T}_{0} \wedge X \wedge X\right)\right) ;
$$


it therefore follows from Lemma 8.5 that

$$
2^{r}\left[U \circ \Sigma^{j} \bar{U} \circ\left(T_{0} \wedge X \wedge X\right) \circ \Sigma^{2 j} U \circ \Sigma^{3 j} \bar{U} \circ\left(\Sigma^{2 j} T_{0} \wedge X \wedge X\right)\right] \simeq_{p} c_{(g \wedge g)^{2}} .
$$

Next observe that

$$
\begin{aligned}
& U \circ \Sigma^{j} \bar{U} \circ\left(T_{0} \wedge X \wedge X\right) \circ \Sigma^{2 j} U \circ \Sigma^{3 j} \bar{U} \circ \Sigma^{2 j}\left(T_{0} \wedge X \wedge X\right) \\
& \quad=U \circ \Sigma^{j} \bar{U} \circ \Sigma^{2 j} U \circ \Sigma^{3 j} \bar{U} \circ\left(T^{\tau \tau} \wedge X \wedge X\right)
\end{aligned}
$$

for some homotopy $T^{\tau \tau}$ from the identity to the permutation $S_{1}^{j} \wedge S_{2}^{j} \wedge S_{3}^{j} \wedge S_{4}^{j} \rightarrow$ $S_{2}^{j} \wedge S_{1}^{j} \wedge S_{4}^{j} \wedge S_{3}^{j}$. Applying Lemmas 8.4 and 8.5, we then have

$$
\begin{aligned}
c_{(g \wedge g)^{2}} \simeq & 2^{r}\left[U \circ \Sigma^{j} \bar{U} \circ \Sigma^{2 j} U \circ \Sigma^{3 j} \bar{U} \circ\left(T^{\tau \tau} \wedge X \wedge X\right)\right] \\
\simeq & 2^{r}\left[U \circ \Sigma^{j} \bar{U} \circ \Sigma^{2 j} U \circ\left(\Sigma^{j} \bar{T}_{0} \wedge X \wedge X\right)\right. \\
& \left.\circ\left(\Sigma^{j} \bar{T}_{0} \wedge X \wedge X\right) \circ \Sigma^{3 j} \bar{U} \circ\left(T^{\tau \tau} \wedge X \wedge X\right)\right] \\
\simeq & 2^{r}\left[U \circ \Sigma^{j} U \circ \Sigma^{2 j} \bar{U} \circ\left(\Sigma^{j} T_{0} \wedge X \wedge X\right) \circ\left(\Sigma^{j} \bar{T}_{0} \wedge X \wedge X\right)\right. \\
& \left.\circ \Sigma^{3 j} \bar{U} \circ\left(T^{\tau \tau} \wedge X \wedge X\right)\right] \\
\simeq & 2^{r}\left[U \circ \Sigma^{j} U \circ \Sigma^{2 j} \bar{U} \circ \Sigma^{3 j} \bar{U} \circ(\sigma \wedge X \wedge X) \circ\left(T^{\tau \tau} \wedge X \wedge X\right)\right],
\end{aligned}
$$

where $\sigma$ denotes the permutation $S_{1}^{j} \wedge S_{2}^{j} \wedge S_{3}^{j} \wedge S_{4}^{j} \rightarrow S_{1}^{j} \wedge S_{3}^{j} \wedge S_{2}^{j} \wedge S_{4}^{j}$. But

$$
(g \wedge g)^{2} \circ(\sigma \wedge X \wedge X)=g^{2} \wedge g^{2}
$$

and $T \equiv \sigma \circ T^{\tau \tau} \circ \sigma$ is a homotopy from the identity to the switch map $S_{1}^{2 j} \wedge S_{2}^{2 j} \rightarrow$ $S_{2}^{2 j} \wedge S_{1}^{2 j} ;$ therefore

$$
c_{g^{2} \wedge g^{2}} \simeq{ }_{p} 2^{r}\left[U^{(2)} \circ \Sigma^{2 j} \bar{U}^{(2)} \circ(T \wedge X \wedge X)\right]
$$

as desired.

This procedure may be iterated. For example, it follows from Eqs. 8.2, 8.4, and Lemma 8.5 that

$$
\begin{aligned}
& 2^{r}\left[U \circ \Sigma^{j} \bar{U} \circ\left(T_{0} \wedge X \wedge X\right) \circ \Sigma^{2 j} \bar{U} \circ \Sigma^{3 j} U \circ \Sigma^{2 j}\left(\bar{T}_{0} \wedge X \wedge X\right) \circ \Sigma^{4 j} U^{(2)}\right. \\
& \left.\quad \circ \Sigma^{6 j} \bar{U}^{(2)} \circ\left(\Sigma^{4 j} T \wedge X \wedge X\right)\right]
\end{aligned}
$$

is path homotopic to the constant loop at $(g \wedge g)^{2} \circ \Sigma^{4 j}\left(g^{2} \wedge g^{2}\right)$. Then apply Eq. 8.3 repeatedly as above to obtain

$$
c_{g^{4} \wedge g^{4}} \simeq p 2^{r}\left[U^{(4)} \circ \Sigma^{4 j} \bar{U}^{(4)} \circ(T \wedge X \wedge X)\right]
$$


for some (other) homotopy $T: I \rightarrow \operatorname{Map}\left(S^{4 j} \wedge S^{4 j}, S^{4 j} \wedge S^{4 j}\right)$ from the identity to the switch map. Continue this process to obtain the desired result for all $m$.

\section{Proof of Lemma 5.8}

We are now ready to complete the proof of Lemma 5.8 by showing that, for appropriate iterates of our original $v_{n}$ self-map, the homotopies appearing in the diagrams 7.2 may be chosen so that the hypotheses of Proposition 7.2 are satisfied.

Suppose that $h$ is an iterate of $g$ such that $j=\operatorname{deg} h$ is even and such that there exists a homotopy $U$ from $h \wedge X$ to $X \wedge h$ such that

$$
\begin{gathered}
(h \wedge X) \circ \Sigma^{j} U \simeq{ }_{p} U \circ\left(\Sigma^{j} h \wedge X\right) \circ(T \wedge X \wedge X) \\
(X \wedge h) \circ \Sigma^{j} U \circ(T(1) \wedge X \wedge X) \simeq{ }_{p} U \circ\left(\Sigma^{j} X \wedge h\right) \circ(T \wedge X \wedge X),
\end{gathered}
$$

where $T: I \rightarrow \operatorname{Map}\left(S^{2 j}, S^{2 j}\right)$ is some homotopy from the identity to the switch map on $S^{j} \wedge S^{j}$. We will show that such a map $h$ allows for the desired compatibility of homotopies. By virtue of Corollary 8.3, this suffices to prove Lemma 5.8.

We now identify the homotopies appearing in Proposition 7.2 applied to the diagrams 7.2. Begin by fixing a natural homotopy $C_{f}$ from $e\left(\Sigma p_{f}\right) \circ e\left(\delta_{f}\right)$ to $\left\langle\Sigma^{k+1} X \wedge \Sigma f\right\rangle \circ e\left(q_{f}\right)$ as in Proposition 5.5, and define

$$
H(r): I \rightarrow \operatorname{Map}^{S}\left(e\left(\Sigma^{r k+1} X \wedge \Sigma^{r k+1} X\right)^{\prime}, e\left(\Sigma^{2}\left(\Sigma^{r k} X \wedge X \vee X \wedge \Sigma^{r k} X\right)\right)\right)
$$

by

$$
H(r)=C_{f^{r}} *\left\langle\Sigma^{2+r k}\left[\left(X \wedge h^{r}\right) \circ \Sigma^{r j} \bar{U}^{(r)}\right] \circ\left(\Sigma^{2} P(r) \wedge X \wedge X\right) \circ q_{f^{r}} .\right.
$$

Here $P(r): I \rightarrow \operatorname{Map}\left(S^{4 r j}, S^{4 r j}\right)$ is any choice of homotopy from the identity to the permutation

$$
S_{1}^{r j} \wedge S_{2}^{r j} \wedge S_{3}^{r j} \wedge S_{4}^{r j} \rightarrow S_{1}^{r j} \wedge S_{3}^{r j} \wedge S_{4}^{r j} \wedge S_{2}^{r j}
$$

$\Sigma^{r k+1} X \wedge \Sigma^{r k+1} X$ is identified with $\Sigma^{2 r k+2} X \wedge X$ via

$$
\Sigma_{1} \Sigma_{2}^{r k} X \wedge \Sigma_{3} \Sigma_{4}^{r k} X=\Sigma_{1} \Sigma_{3} \Sigma_{2}^{r k} \Sigma_{4}^{r k} X \wedge X
$$

and we include $\Sigma^{2+r k}(X \wedge X)$, the target of

$$
\Sigma^{2+r k}\left[\left(X \wedge h^{r}\right) \circ \Sigma^{r j} \bar{U}^{(r)}\right] \circ\left(\Sigma^{2} P(r) \wedge X \wedge X\right)
$$

in $\Sigma^{2}\left(\Sigma^{r k} X \wedge X \vee X \wedge \Sigma^{r k} X\right)$ in the usual way. Define the homotopies $H_{1}$ and $H_{2}$ by $H_{1}=H(2)$ and $H_{2}=H(1)$. The homotopy

$$
K: I \rightarrow \operatorname{Map}^{S}\left(e\left(\Sigma^{k+1} X \wedge \Sigma^{k+1} X\right), e\left(\Sigma^{2}\left(\Sigma^{k} X \wedge X \vee X \wedge \Sigma^{k} X\right)\right)\right)
$$


may be defined by

$$
K=\left\langle\Sigma^{2+k}\left[(h \wedge X) \circ \Sigma^{j} U\right] \circ\left(\Sigma^{2} T_{0} \wedge X \wedge X\right)\right\rangle,
$$

where $\Sigma^{k+1} X \wedge \Sigma^{k+1} X$ is identified with $\Sigma^{2 k+2} X \wedge X$ as in Eq. 9.2,

$$
T_{0}: I \rightarrow \operatorname{Map}\left(S^{4 j}, S^{4 j}\right)
$$

is any choice of homotopy from the identity to the conjugation

$$
S_{1}^{j} \wedge S_{2}^{j} \wedge S_{3}^{j} \wedge S_{4}^{j} \rightarrow S_{1}^{j} \wedge S_{3}^{j} \wedge S_{2}^{j} \wedge S_{4}^{j},
$$

and we include $\Sigma^{2+k} X \wedge X$, the target of $\Sigma^{2+k}\left[(h \wedge X) \circ \Sigma^{j} U\right] \circ\left(\Sigma^{2} T_{0} \wedge X \wedge X\right)$, in $\Sigma^{2}\left(\Sigma^{k} X \wedge X \vee X \wedge \Sigma^{k} X\right)$ as above. Finally, observe that

$$
\begin{aligned}
\gamma_{1} & =e\left(\Sigma^{k+1} f \wedge \Sigma^{k+1} f\right)^{\prime} \\
i_{1} & =e\left(\Sigma^{k+1} f \wedge \Sigma^{k+1} f\right) \circ e\left(q_{f^{2}}\right) \\
d & =e\left(\Sigma^{2}\left(\Sigma^{k} f \wedge X \vee X \wedge \Sigma^{k} f\right)\right) .
\end{aligned}
$$

Since $C_{f} \circ \gamma_{1}=d \circ C_{f^{2}}$ by naturality, the hypotheses of Proposition 7.2 are satisfied for diagrams 7.2 provided that the map

$$
B: \dot{\Delta}^{2} \rightarrow \operatorname{Map}^{S}\left(\Sigma^{4 k} X \wedge X, \Sigma^{k} X \wedge X\right)
$$

given by

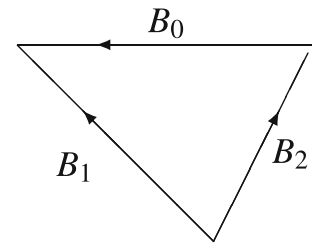

extends over $\Delta^{2}$, where

$$
\begin{aligned}
& B_{0}=\Sigma^{k}\left[(h \wedge X) \circ \Sigma^{j} \bar{U}\right] \circ\left(\bar{T}_{0} \wedge X \wedge X\right) \circ\left(\Sigma^{k} f \wedge \Sigma^{k} f\right) \\
& B_{1}=\Sigma^{k}\left[(f \wedge f) \circ \Sigma^{2 k} \bar{U}^{(2)}\right] \circ(P(2) \wedge X \wedge X) \\
& B_{2}=\Sigma^{k}\left[(X \wedge h) \circ \Sigma^{j} \bar{U}\right] \circ(P(1) \wedge X \wedge X) \circ\left(\Sigma^{k} f \wedge \Sigma^{k} f\right) .
\end{aligned}
$$

Using the relations 9.1, we have

$$
\begin{aligned}
& B_{0} \simeq_{p} \Sigma^{k}\left[\bar{U} \circ\left(\Sigma^{j} h \wedge X\right)\right] \circ\left(\Sigma^{k} f \wedge \Sigma^{k} f\right) \circ\left(S_{0} \wedge X \wedge X\right) \\
& B_{1} \simeq p \Sigma^{k} \bar{U}^{(2)} \circ\left(\Sigma^{k} f \wedge \Sigma^{k} f\right) \circ\left(\bar{S}_{1} \wedge X \wedge X\right) \\
& B_{2} \simeq_{p} \Sigma^{k}\left[(X \wedge h) \circ \Sigma^{j} \bar{U}\right] \circ\left(\Sigma^{k} f \wedge \Sigma^{k} f\right) \circ\left(S_{2} \wedge X \wedge X\right),
\end{aligned}
$$


where each $S_{i}: I \rightarrow \operatorname{Map}\left(S^{8 j}, S^{8 j}\right)$ is a homotopy from a permutation $\sigma_{i} \in \Sigma_{8}$ to $\sigma_{i+1} \in \Sigma_{8}$ with $\sigma_{3}=\sigma_{0}$.

Now $\sigma_{2}=i d$ and thus $S_{2} * S_{0} * S_{1} \in \pi_{1}\left(\operatorname{Map}_{\sigma_{2}}\left(S^{8 j}, S^{8 j}\right)\right.$ is path homotopic to $\omega \wedge S^{6 j}$ for some $\omega \in \pi_{1}\left(\operatorname{Map}_{i d}\left(S^{2 j}, S^{2 j}\right)\right)$. Of course, $\omega \simeq_{p} \bar{\omega}$, and if we replace $P(1)$ above by $\left(\omega \wedge S^{2 j}\right) * P(1)$, then we obtain $S_{2} * S_{0} * S_{1} \simeq p c_{i d}$. We also have that

$$
\bar{U}^{(2)} \simeq_{p}\left[(X \wedge h) \circ \Sigma^{j} \bar{U}\right] *\left[\bar{U} \circ\left(\Sigma^{j} h \wedge X\right)\right]
$$

hence it follows from 9.4 that $B_{2} * B_{0} \simeq{ }_{p} B_{1}$. This proves that $B$ extends over $\Delta^{2}$ as desired.

\section{Appendix 1: Proof of Theorem 1.4}

In this section, we will be working entirely within the stable category, and we will write $D_{j}(X)$ for $E \Sigma_{j} \ltimes \Sigma_{j} \Gamma X^{(j)}$, where $\Gamma X$ is, as before, a CW-approximation to $X$. If $f: X \rightarrow Y$, we will write $D_{p}(f)$ for the evident map from $D_{p}(X)$ to $D_{p}(Y)$.

We begin with a couple of observations. First, if $M\left(p^{i}\right)$ has a $p$-fold multiplication with unit, then the unit map must be just the usual inclusion of the bottom cell. Second, since $\left[E \Sigma_{p}^{N} \ltimes \Sigma_{p} M\left(p^{i}\right)^{(p)}, M\left(p^{i}\right)\right]$ is finite for each $N$, it suffices to show that no such multiplication on $M\left(p^{i}\right)$ extends over $D_{p}\left(M\left(p^{i}\right)\right)$.

Our proof follows an argument of Nishida used in his proof that elements of positive degree in $\pi_{*} S^{0}$ are nilpotent [9]. This work has been formulated in more modern language in [1], and this is the reference we will use.

Let $\tau_{p}(X): D_{p}(X) \rightarrow X^{(p)}$ be the map defined in [1, Chapter 2, Definition 1.4], and let $\iota_{p}(X)$ be the composition in Eq. 1.2. When no confusion will result, we will write $\tau_{p}(X)$ and $\iota_{p}(X)$ as $\tau_{p}$ and $\iota_{p}$ respectively. By [1, Chapter 2, Corollary 1.8], we have that $D_{p}\left(p^{i}\right): D_{p}(X) \rightarrow D_{p}(X)$ decomposes as

$$
D_{p}\left(p^{i}\right)=p^{i} \lambda+u p^{i-1} \iota_{p} \tau_{p}
$$

for some $\lambda: D_{p}(X) \rightarrow D_{p}(X)$ and unit $u$ in $\mathbb{Z}_{(p)}$.

If $M\left(p^{i}\right)$ has a ring spectrum structure, then $p^{i}: M\left(p^{i}\right) \rightarrow M\left(p^{i}\right)$ is trivial; it therefore follows from 10.1 that, if the multiplication on $M\left(p^{i}\right)$ extends to a map $\xi: D_{p}\left(M\left(p^{i}\right)\right) \rightarrow M\left(p^{i}\right)$, then the composition

$$
D_{p}\left(M\left(p^{i}\right)\right) \stackrel{\tau_{p}}{\longrightarrow} M\left(p^{i}\right)^{(p)} \stackrel{\mu}{\longrightarrow} M\left(p^{i}\right)
$$

is annihilated by $p^{i-1}$. Here, of course, $\mu$ is the $p$-fold multiplication on $M\left(p^{i}\right)$.

Now choose an element $\alpha \in \pi_{*} S^{0}$ such that $p^{i-1} \alpha$ is not divisible by $p^{i}$. Such an $\alpha$ clearly exists; for example, it may be chosen to be in the image of the $J$ homomorphism. By the Kahn-Priddy theorem (see[1, Chapter 2, Theorem 2.8]), there exists $h \in \pi_{*}\left(D_{p}\left(S^{0}\right)\right)$ such that $\left(\tau_{p}\right)_{*} h=\alpha$. Let

$$
\bar{h}=D_{p}(\eta)_{*} h \in \pi_{*} D_{p}\left(M\left(p^{i}\right)\right),
$$


where $\eta: S^{0} \rightarrow M\left(p^{i}\right)$ is the unit. Then

$$
\mu_{*} \tau_{p}\left(M\left(p^{i}\right)\right)_{*} \bar{h}=\mu_{*} \eta_{*}^{(p)} \tau_{p}\left(S^{0}\right)_{*} h=\mu_{*} \eta_{*}^{(p)} \alpha=\eta_{*} \alpha \in \pi_{*} M\left(p^{i}\right)
$$

But $p^{i-1} \eta_{*} \alpha \neq 0$, a contradiction.

\section{Appendix 2: Further directions}

The reader may have noticed that our proof of Theorem 1.1 made little use of properties of the multiplication on $X$; instead, most of our work involved a detailed study of the $v_{n}$ self-map $g$. This study, in turn, had at its essence an understanding of the centrality properties of $v_{n}$ self-maps. In this Appendix, we will describe an alternative approach to organizing the proof of Theorem 1.1, one that more explicitly indicates what is required of $g$. We will then use this approach to suggest a program for proving the more general question involving actions of finite skeleta of $E_{\infty}$ operads. In addition, we will see how this approach might be used to prove other desired properties of a multiplication, such as the derivation property used by Oka. Finally, we will suggest a version of centrality of $v_{n}$ self-maps applicable to certain diagrams of type $n$ spectra commutative up to (a finite level of) higher homotopy. This notion appears to be an important ingredient in any attempt to achieve our program. While much of this Appendix is speculative, we hope that it illuminates some previously unexplored directions.

A key piece in our program is a generalization of Proposition 2.3 to extended powers. We begin with some notation. If $X, Y$ are $S$-modules and $f: Y \rightarrow X$, filter $C(f)$ by $F_{0} C(f)=X$ and $F_{1} C(f)=C(f)$. This filtration induces a filtration on $C(f)^{(j)}$ by

$$
F_{i}\left(C(f)^{(j)}\right)=\bigcup_{i_{1}+\cdots+i_{j} \leq i}\left(F_{i_{1}} C(f) \wedge \cdots \wedge F_{i_{j}} C(f)\right)
$$

and a filtration on $e_{E, j}\left(C(f)^{(j)}\right)$ by

$$
F_{i} e_{E, j}\left(C(f)^{(j)}\right)=e_{E, j}\left(F_{i}\left(C(f)^{(j)}\right)\right)
$$

In addition, write

$$
Q_{i}\left(C(f)^{(j)}\right)=\frac{F_{i}\left(C(f)^{(j)}\right)}{F_{i-1}\left(C(f)^{(j)}\right)} ;
$$

then

$$
e_{E, j} Q_{i}\left(C(f)^{(j)}\right)=\frac{F_{i} e_{E, j}\left(C(f)^{(j)}\right)}{F_{i-1} e_{E, j}\left(C(f)^{(j)}\right)} .
$$


In particular, if $t: Y \rightarrow X$ is the trivial map,

$$
e_{E, j}\left(C(t)^{(j)}\right)=e_{E, j}\left((X \vee \Sigma Y)^{(j)}\right)=\bigvee_{0 \leq i \leq j} e_{E, j}\left(Q_{i}(X \vee \Sigma Y)^{(j)}\right),
$$

and $Q_{i}(X \vee \Sigma Y)^{(j)}$ is the summand of $(X \vee \Sigma Y)^{(j)}$ with exactly $i$ factors of $\Sigma Y$. Note further that $Q_{i}\left(C(f)^{(j)}\right)=Q_{i}(X \vee \Sigma Y)^{(j)}$ as $S$-modules with a $\Sigma_{j}$-action, so that $e_{E, j}\left(Q_{i} C(f)^{(j)}\right)=e_{E, j}\left(Q_{i}(X \vee \Sigma Y)^{(j)}\right)$. We will mostly be interested in the case where $Y=\Sigma^{k} X$.

Now specialize to $j=2$, and assume that $g: \Sigma^{|g|} X \rightarrow X$ is a $v_{n}$ self-map. Of course

$$
F_{0} e\left(C(g)^{(2)}\right)=e(X \wedge X)=F_{0} e\left(X \vee \Sigma^{|g|+1} X\right)^{(2)}
$$

moreover, if $r: \Sigma^{|g|} Y \rightarrow Y$ is another $v_{n}$ self-map-inducing multiplication by the same power of $p$ on rational homology as $g$ if $n=0$ - it follows from Proposition 5.1 together with Proposition 2.3 that there exists $N$ such that

$$
F_{1} e\left(C\left(g^{m N}\right)^{(2)}\right) \wedge C\left(r^{(m N)}\right) \simeq F_{1} e\left(\left(X \vee \Sigma^{m N|g|+1} X\right)^{(2)}\right) \wedge C\left(r^{(m N)}\right)
$$

for any positive integer $m$. If in addition $E$ is finite, then Corollary 5.10 applies and implies — with $N$ chosen larger if necessary-that

$$
e\left(\delta_{f}\right): e\left(\Sigma^{k+1} X \wedge \Sigma^{k+1} X\right)^{\prime} \rightarrow \Sigma F_{1} e\left(C(f)^{(2)}\right)
$$

factors as a composition

$$
e\left(\Sigma^{k+1} X \wedge \Sigma^{k+1} X\right)^{\prime} \stackrel{e\left(\Sigma^{j+1} h \wedge \Sigma^{j+1} h\right) \circ e\left(q_{f}\right)}{\longrightarrow} e\left(\Sigma^{j+1} X \wedge S^{j+1} X\right) \rightarrow \Sigma F_{1} e\left(C(f)^{(2)}\right)
$$

Here $f=h^{2}, h=g^{m N / 2}$, and $k, j$ are the degrees of $f$ and $h$ respectively. Choosing $N$ perhaps still larger, Proposition 2.3 then implies that $e\left(\delta_{g^{m N}}\right) \wedge C\left(r^{m N}\right) \simeq *$ and therefore

$$
\begin{aligned}
e\left(C\left(g^{m N}\right)^{(2)}\right) \wedge C\left(r^{m N}\right) & \simeq\left[F_{1} e\left(C\left(g^{m N}\right)^{(2)}\right) \vee e\left(\Sigma^{k+1} X \wedge \Sigma^{k+1} X\right)\right] \wedge C\left(r^{m N}\right) \\
& \simeq\left[F_{1} e\left(X \vee \Sigma^{k+1} X\right)^{(2)} \vee e\left(\Sigma^{k+1} X \wedge \Sigma^{k+1} X\right)\right] \wedge C\left(r^{m N}\right) \\
& \simeq e\left(X \vee \Sigma^{k+1} X\right)^{(2)} \wedge C\left(r^{m N}\right) .
\end{aligned}
$$

Of course, Proposition 2.3 also implies that

$$
C\left(g^{m N}\right) \wedge C\left(r^{m N}\right) \simeq\left(X \vee \Sigma^{k+1} X\right) \wedge C\left(r^{m N}\right)
$$


furthermore, the equivalences 11.2 and 11.3 may be chosen so that

$$
\begin{aligned}
& C\left(g^{m N}\right) \wedge C\left(r^{m N}\right) \quad \stackrel{\simeq}{\longrightarrow}\left(X \vee \Sigma^{k+1} X\right) \wedge C\left(r^{m N}\right) \\
& \downarrow \eta \wedge C\left(r^{m N}\right) \quad \downarrow \eta \wedge C\left(r^{m N}\right) \\
& e\left(C\left(g^{m N}\right)^{(2)}\right) \wedge C\left(r^{m N}\right) \stackrel{\simeq}{\longrightarrow} e\left(\left(X \vee \Sigma^{k+1} X\right)^{(2)}\right) \wedge C\left(r^{m N}\right)
\end{aligned}
$$

commutes up to homotopy. Here we are using the following convention: If $Z$ is any $S$-module with a map $\eta: S^{0} \rightarrow Z$, we also write $\eta$ for the composition

$$
Z=Z \wedge S^{0} \stackrel{Z \wedge \eta}{\longrightarrow} Z \wedge Z=\mathbb{Z} /(2) \ltimes_{\mathbb{Z} /(2)}(Z \wedge Z) \longrightarrow e(Z \wedge Z) .
$$

Observe that if $X$ has an $E$-commutative ring spectrum structure $\xi_{X}: e(X \wedge X) \rightarrow$ $X$, then so does $X \vee \Sigma^{k+1} X$. Indeed, use the splitting 11.1 and define $\xi_{X \vee \Sigma^{k+1} X}$ by $\xi_{X \vee \Sigma^{k+1} X}\left|e\left(Q_{0}\left(X \vee \Sigma^{k+1} X\right)^{(2)}\right)=\xi_{X}, \xi_{X \vee \Sigma^{k+1} X}\right| e\left(Q_{2}\left(X \vee \Sigma^{k+1} X\right)^{(2)}\right)=*$, and $\xi_{X \vee \Sigma^{k+1} X} \mid e\left(Q_{1}\left(X \vee \Sigma^{k+1} X\right)^{(2)}\right)$ to be the map $\xi_{X, k+1}$ of Proposition 5.2, followed by the inclusion $\Sigma^{k+1} X \rightarrow X \vee \Sigma^{k+1} X$.

Since $D C\left(g^{m N}\right) \simeq \Sigma^{-1} D\left(g^{m N}\right)$, we may now use the equivalences 11.2 and 11.3 to write $\xi_{X \vee \Sigma^{k+1} X} \wedge D C\left(g^{m N}\right)$ as a map

$$
e\left(C\left(g^{m N}\right)^{(2)}\right) \wedge D C\left(g^{m N}\right) \longrightarrow C\left(g^{m N}\right) \wedge D C\left(g^{m N}\right) .
$$

We may then form the composition

$$
e\left(C\left(g^{m N}\right)^{(2)}\right) \wedge D C\left(g^{m N}\right) \rightarrow C\left(g^{m N}\right) \wedge D C\left(g^{m N}\right) \stackrel{\varepsilon}{\rightarrow} S^{0},
$$

where $\varepsilon$ is adjoint to the identity map. Taking the adjoint of this compostion yields a map

$$
\xi_{C\left(g^{m N}\right)}: e\left(C\left(g^{m N}\right)^{(2)}\right) \longrightarrow C\left(g^{m N}\right)
$$

and one can check, using the commutative diagram 11.4, that this map is an $E$ commutative ring spectrum structure on $C\left(g^{m N}\right)$.

Moreover, the equivalences of 11.2 and 11.3 are filtration preserving $-C\left(r^{n M}\right)$ is given the trivial filtration-and are in fact the identity on the associated quotients. This observation applied to $F_{0}$ implies that $\xi_{C\left(g^{m N}\right)}$ extends the $E$-commutative ring spectrum structure on $X$.

The approach just outlined suggests a way to attack the general problem of constructing higher multiplicative structures on the cofiber of a $v_{n}$ self-map. Begin by observing that, just as before, any map $\xi_{X}: e_{E, j}\left(X^{(j)}\right) \rightarrow X$ induces a map $\xi_{X \vee \Sigma^{k+1} X}: e_{E, j}\left(\left(X \vee \Sigma^{k+1} X\right)^{(j)}\right) \rightarrow X$ which is trivial on $e_{E, j}\left(Q_{i}\left(X \vee \Sigma^{k+1} X\right)^{(j)}\right)$ for $i>1$. In this way, any higher multiplicative structure on $X$ induces the same sort of multiplicative structure on $X \vee \Sigma^{k+1} X$. Now let $g: \Sigma^{|g|} X \rightarrow X$ and $r: \Sigma^{|g|} Y \rightarrow Y$ 
be $v_{n}$ self-maps as before. We hope to find $N>0$ such that, for each $m>0$, there are filtration preserving equivalences

$$
\left[E \Sigma_{j}^{i} \ltimes_{\Sigma_{j}} C\left(g^{m N}\right)^{(j)}\right] \wedge C\left(r^{m N}\right) \simeq\left[E \Sigma_{j}^{i} \ltimes_{\Sigma_{j}}\left(X \vee \Sigma^{k+1} X\right)^{(j)}\right] \wedge C\left(r^{m N}\right)
$$

$k=m N|g|$, valid for all $j \leq M$ and $i \leq k_{j}$. (Equivalence 11.3 is the $j=1$ case). In addition, we should require that these equivalences become the identity upon passing to the quotients of the filtration and that, if $M>2$, these equivalences are compatible with the inclusions $E \Sigma_{j}^{i} \rightarrow E \Sigma_{j}^{i+1}$. If $X$ has a higher multiplicative structure with structure maps $\xi_{j, X}: E \Sigma_{j}^{k_{j}} \ltimes X^{(j)} \rightarrow X$, then we may, as before, use 11.7 and 11.3 to write $\xi_{j, X \vee \Sigma^{k+1} X} \wedge D C\left(g^{m N}\right)$ as a map

$$
\left[E \Sigma_{j}^{k_{j}} \ltimes \Sigma_{j} C\left(g^{m N}\right)^{(j)}\right] \wedge D C\left(g^{m N}\right) \longrightarrow C\left(g^{m N}\right) \wedge D C\left(g^{m N}\right),
$$

which, when composed with the adjoint $\varepsilon: C\left(g^{m N}\right) \wedge D C\left(g^{m N}\right) \rightarrow S^{0}$ of the identity, yields a map

$$
\left[E \Sigma_{j}^{k_{j}} \ltimes_{\Sigma_{j}} C\left(g^{m N}\right)^{(j)}\right] \wedge D C\left(g^{m N}\right) \longrightarrow S^{0}
$$

The adjoint of this map is defined to be

$$
\xi_{j, C\left(g^{m N}\right)}: E \Sigma_{j}^{k_{j}} \ltimes_{\Sigma_{j}} C\left(g^{m N}\right)^{(j)} \longrightarrow C\left(g^{m N}\right) .
$$

The idea then is that appropriate compatibility properties of the equivalences 11.7 ought to reduce the problem of proving the required relationships among the $\xi_{j, C\left(g^{m N}\right)}$ 's to observing that they hold for the $\xi_{j, X \vee \Sigma^{k+1} X}$ 's. For example, the diagram 11.4 is the relevant diagram to establish that the multiplication on $C\left(g^{m N}\right)$ is unital (in the stable category).

Although we are not yet able to write down all of the compatibility diagrams, we can give a non-trivial related example, arising from Oka's work on explicit $v_{n}$ self-maps. Recall that in this work, it is important for the multiplication on $C\left(g^{m N}\right)$ to have the property that the map

$$
d_{m N}^{g}: C\left(g^{m N}\right) \longrightarrow \Sigma^{m N|g|+1} X \longrightarrow \Sigma^{m N|g|+1} C\left(g^{m N}\right)
$$

is a derivation. But the map

$$
d_{m N}^{t}: X \vee \Sigma^{k+1} X \longrightarrow \Sigma^{k+1} X \longrightarrow \Sigma^{k+1}\left(X \vee \Sigma^{k+1} X\right)
$$

is certainly a derivation; one might then expect that the desired property of $d_{m N}^{g}$ is a consequence of properties of the equivalences 11.2 and 11.3. This is indeed the case. 
Let $D_{m N}^{g}$ and $D_{m N}^{t}$ denote the compositions

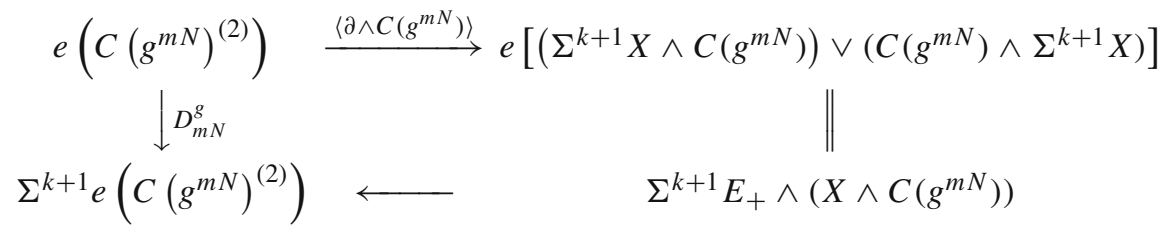

and

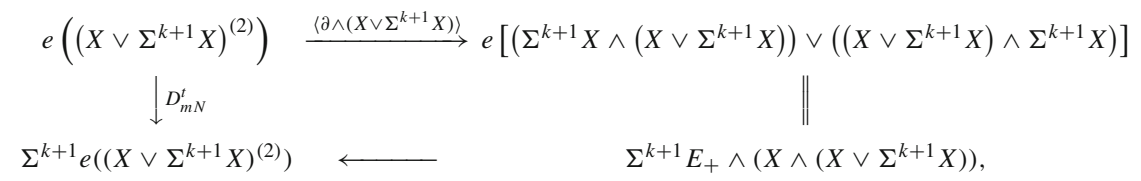

and suppose that, with the equivalences 11.2 and 11.3, the following diagrams are homotopy commutative:

$$
\begin{aligned}
& e\left(C\left(g^{m N}\right)^{(2)}\right) \wedge C\left(r^{m N}\right) \quad \stackrel{\simeq}{\longrightarrow} e\left(\left(X \vee \Sigma^{k+1} X\right)^{(2)}\right) \wedge C\left(r^{m N}\right) \\
& \downarrow D_{m N}^{g} \wedge C\left(r^{m N}\right) \quad \downarrow D_{m N}^{t} \wedge C\left(r^{m N}\right) \\
& \Sigma^{k+1} e\left(C\left(g^{m N}\right)^{(2)}\right) \wedge C\left(r^{m N}\right) \simeq \Sigma^{k+1} e\left(\left(X \vee \Sigma^{k+1} X\right)^{(2)}\right) \wedge C\left(r^{m N}\right) \\
& C\left(g^{m N}\right) \wedge C\left(r^{m N}\right) \stackrel{\simeq}{\longrightarrow}\left(X \vee \Sigma^{k+1} X\right) \wedge C\left(r^{m N}\right) \\
& \downarrow d_{m N}^{g} \wedge C\left(r^{m N}\right) \quad \downarrow d_{m N}^{t} \wedge C\left(r^{m N}\right) \\
& \Sigma^{k+1} C\left(g^{m N}\right) \wedge C\left(r^{m N}\right) \stackrel{\simeq}{\longrightarrow} \Sigma^{k+1}\left(X \vee \Sigma^{k+1} X\right) \wedge C\left(r^{m N}\right) \\
& e\left(C\left(g^{m N}\right)^{(2)}\right) \wedge C\left(r^{m N}\right) \stackrel{\simeq}{\longrightarrow} e\left(\left(X \vee \Sigma^{k+1} X\right)^{(2)}\right) \wedge C\left(r^{m N}\right) \\
& \downarrow e\left(C\left(g^{m N}\right)^{(2)}\right) \wedge d_{m N}^{r} \quad \downarrow(i d) \wedge d_{m N}^{r} \\
& \Sigma^{k+1} e\left(C\left(g^{m N}\right)^{(2)}\right) \wedge C\left(r^{m N}\right) \simeq \Sigma^{k+1} e\left(\left(X \vee \Sigma^{k+1} X\right)^{(2)}\right) \wedge C\left(r^{m N}\right)
\end{aligned}
$$

$$
\begin{aligned}
& C\left(g^{m N}\right) \wedge C\left(r^{m N}\right) \stackrel{\simeq}{\longrightarrow}\left(X \vee \Sigma^{k+1} X\right) \wedge C\left(r^{m N}\right) \\
& \downarrow C\left(g^{m N}\right) \wedge d_{m N}^{r} \quad \downarrow\left(X \vee \Sigma^{k+1} X\right) \wedge d_{m N}^{r}
\end{aligned}
$$

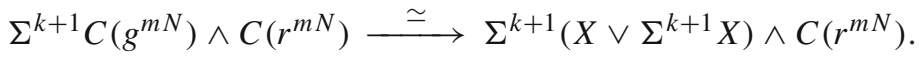


Then one can show that $d_{m N}^{g}$ is a derivation for the multiplication $\xi_{C\left(g^{m N}\right)}$ defined as the adjoint of 11.6. (Actually, the above diagrams need only commute for $r^{m N}=D g^{m N}$ ). Observe that the homotopy commutativity of diagram 11.9 is immediate from our construction of the equivalence 11.3 but that the homotopy commutativity of the others is not obvious.

How then might one establish the equivalences 11.7 together with the requisite compatibility properties? In our view, an important ingredient will be understanding $v_{n}$ self-maps on diagrams on type $n$ spectra. To be sure, more may be required, but since this idea seems to be of independent interest, we will explore it in some detail now. Let us begin by making this notion a little more precise.

Let $\mathscr{M}_{+}$denote the category whose objects are $S$-modules; the set of morphisms between $X$ and $Y$ is the disjoint union of the $S$-module maps from $\Sigma^{k} X$ to $Y$ as $k$ ranges over the nonnegative integers. If $\mathbf{I}$ is a small category, we may define the notion of an $M$-homotopy commutative $\mathbf{I}$-diagram $\mathbf{F}$ in $\mathscr{M}_{+}$for any positive integer $M$ (cf [3]). For example, a 1-homotopy commutative diagram is just a pair of maps which sends each object $i$ in $\mathbf{I}$ to an object $F(i)$ in $\mathscr{M}_{+}$and each morphism $f: i \rightarrow j$ in $\mathbf{I}$ to a morphism $F(f): F(i) \rightarrow F(j)$ in $\mathscr{M}_{+}$such that $F\left(i d_{i}\right)=i d_{F(i)}$ and $F(g) \circ F(f) \simeq$ $F(g \circ f)$ whenever $g: j \rightarrow k$. A 2-homotopy commutative diagram consists of all the data for a 1-homotopy commutative diagram together with homotopies $H(g, f)$ from $F(g) \circ F(f)$ to $F(g \circ f)$ such that

$$
(F(h) \circ H(g, f)) * H(h, g \circ f) \simeq_{p}(H(h, g) \circ F(f)) * H(h \circ g, f)
$$

in the appropriate space of maps for any composable triple of morphisms in I. In addition, we require that $H(i d, g)$ and $H(f, i d)$ are the constant homotopies. This process may be continued by the introduction of higher and higher homotopies to define an $M$-homotopy commutative diagram for general $M$.

If $\mathbf{F}$ and $\mathbf{F}^{\prime}$ are $M$-homotopy commutative I-diagrams, a map $\mathbf{w}: \mathbf{F} \rightarrow \mathbf{F}^{\prime}$ consists of a morphism $w(i): F(i) \rightarrow F^{\prime}(i)$ for each object $i$ in $\mathbf{I}$ together with (higher) homotopies expressing the compatibility of these morphisms with the (higher) homotopies occuring in $\mathbf{F}$ and $\mathbf{F}^{\prime}$. Explicitly, if $M=1$, this amounts to requiring that $F^{\prime}(f) w(i) \simeq w(j) F(f)$ for all $f: i \rightarrow j$ in $\mathbf{I}$. If $M=2$, we must in addition specify a homotopy $G_{w}(f)$ from $F^{\prime}(f) w(i)$ to $w(j) F(f)$-with $G_{w}(i d)$ the constant homotopy-such that

$$
\begin{aligned}
& \left(F^{\prime}(g) \circ G_{w}(f)\right) *\left(G_{w}(g) \circ F(f)\right) \simeq p \\
& \quad\left(H^{\prime}(g, f) \circ w(i)\right) * G_{w}(g \circ f) *(w(k) \circ \overline{H(g, f)})
\end{aligned}
$$

in $\operatorname{Map}^{S}\left(F(i), F^{\prime}(k)\right)$ whenever $f: i \rightarrow j$ and $g: j \rightarrow k$ in $\mathbf{I}$.

We may also talk about homotopies between maps. A homotopy $\mathbf{U}$ from $\mathbf{w}: \mathbf{F} \rightarrow \mathbf{F}^{\prime}$ to $\mathbf{v}: \mathbf{F} \rightarrow \mathbf{F}^{\prime}$ is a collection of homotopies $U(i)$ from $w(i)$ to $v(i)$ for each object $i$ in I together with higher homotopies expressing certain compatibility conditions. For the lowest values of $M$, the conditions are again easy to describe. If $M=1$, nothing more of the $U(i)$ 's is required, and if $M=2$, we require, for each $f: i \rightarrow j$ in $\mathbf{I}$, a 
path homotopy $L(f)$ from $G_{w}(f) *(U(j) \circ F(f))$ to $\left(F^{\prime}(f) \circ U(i)\right) * G_{v}(f)$, with, once again, $L\left(i d_{i}\right)$ the constant homotopy.

Finally, we will sometimes wish to consider $M$-homotopy commutative diagrams as $N$-homotopy commutative diagrams for $N \leq M$ by neglect of structure. Maps between such $N$-homotopy commutative diagrams will be referred to as $N$-homotopy commutative maps and homotopies between them as $N$-homotopies.

Now suppose that $\mathbf{I}$ has only a finite number of objects, and let $\mathbf{F}$ be an $M$-homotopy commutative diagram of type $n$; that is, each $F(i)$ is a finite $p$-local CW $S$-module of type $n$. A $v_{n}$ self-map on $\mathbf{F}$ is a map $\mathbf{v}: \mathbf{F} \rightarrow \mathbf{F}$ such that $v(i): F(i) \rightarrow F(i)$ is a $v_{n}$ self-map for all $i$. (Recall that a map $v(i): F(i) \rightarrow F(i)$ in $\mathscr{M}_{+}$is a map $v(i): \Sigma^{k(i)} F(i) \rightarrow F(i)$ of $S$-modules for some $k(i) \geq 0$; we further assume that $k(i)$ is independent of $i$ when discussing $v_{n}$ self-maps. If $n=0$, we assume that each $v(i)$ induces multiplication by the same power of $p$ on rational homology). If $M=1$, it follows immediately from nilpotence technology that there exists a $v_{n}$ self-map $\mathbf{v}: \mathbf{F} \rightarrow \mathbf{F}$ and that, if $\mathbf{w}: \mathbf{F} \rightarrow \mathbf{F}$ is another $v_{n}$ self-map, then $\mathbf{v}^{r}$ is homotopic to $\mathbf{w}^{s}$ for some $r, s>0$. One might expect that such a result should hold for $M>1$ as well.

Pre-Theorem Suppose that $\mathbf{v}: \mathbf{F} \rightarrow \mathbf{F}$ is a $(M-1)$-homotopy commutative $v_{n}$ self-map. Then there exists such that $\mathbf{v}^{s}$ may be extended to an $M$-homotopy commutative map. If $\mathbf{v}$ and $\mathbf{w}$ are $M$-homotopy commutative $v_{n}$ self-maps and $\mathbf{U}$ is a $(M-1)$-homotopy between them, then there exists $s$ such that $\mathbf{U}^{(s)}$ may be extended to an $M$-homotopy between $\mathbf{v}^{s}$ and $\mathbf{w}^{s}$.

Although we are only able to verify this statement for $M=2$-with an additonal restriction if $n=0$-we have labeled it a "Pre-Theorem" because we believe it is undoubtedly true and that the method of proof in the $M=2$ case should generalize: the only issue is how to organize all of the higher order information in a manageable way. We should also observe here that the compositions above involve "compositions" of all of the higher order information and that has to made sense of as well. We hope to be able to turn this Pre-Theorem into a Theorem in a later paper, but for now, we will content ourselves with presenting an outline of some of its applications to our program.

We begin by observing that a consequence of this Pre-Theorem has already been used. The major technical result needed to establish the splitting 11.2 was Corollary 8.3, and this result may also be interpreted as follows. Using the notation of Sect. 8, let $g: \Sigma^{j} X \rightarrow X$ be a $v_{n}$ self-map such that $g \wedge X$ is homotopic to $X \wedge g$. Let $\mathbf{I}$ be the free monoid on one generator $d$ with object $*$, and let $\mathbf{F}$ be the $\mathbf{I}$-diagram with $F(*)=X \wedge X$ and $F(d)=g \wedge X$. Then $g \wedge X$ is a $v_{n}$ self-map of $\mathbf{F}$, and if $j$ is even, $X \wedge g$ is a 2homotopy commutative $v_{n}$ self-map of $\mathbf{F}$ as well. (The homotopy $G_{X \wedge g}\left(d^{S}\right)$ is defined using an appropriate homotopy between the identity and the permutation map $S_{1}^{s j} \wedge$ $S_{2}^{j} \rightarrow S_{2}^{j} \wedge S_{1}^{s j}$ ). Now let $U$ be a homotopy from $g \wedge X$ to $X \wedge g$. By the Pre-Theorem, there exists an $N$ such that $U^{(N)}$ extends to a 2-homotopy and this fact implies that

$$
\begin{aligned}
(g \wedge X) \circ \Sigma^{j} U^{(N)} & \simeq p\left[U^{(N)} \circ \Sigma^{N j}(g \wedge X)\right] * \overline{G_{X \wedge g^{N}}(d)} \\
& \simeq p\left[U^{(N)} \circ \Sigma^{N j}(g \wedge X)\right] *\left[\left(X \wedge g^{N}\right)\right. \\
& \left.\circ \Sigma^{N j}(g \wedge X) \circ(T \wedge X \wedge X)\right],
\end{aligned}
$$


where $T$ is some homotopy from the identity to the permutation map $S_{1}^{j} \wedge S_{2}^{N j} \rightarrow$ $S_{2}^{N j} \wedge S_{1}^{j}$. But

$$
\begin{aligned}
& {\left[U^{(N)} \circ \Sigma^{N j}(g \wedge X)\right] *\left[\left(X \wedge g^{N}\right) \circ \Sigma^{N j}(g \wedge X) \circ(T \wedge X \wedge X)\right]} \\
& \quad \simeq{ }_{p} U^{(N)} \circ \Sigma^{N j}(g \wedge X) \circ(T \wedge X \wedge X),
\end{aligned}
$$

and putting these two homotopies together is easily seen to imply Corollary 8.3.

Although we will not give details here, we can also use the essential uniqueness up to 2-homotopy of $v_{n}$ self-maps of I-diagrams to prove Proposition 2.3 and gain some understanding of the null homotopy. (As above $\mathbf{I}$ is the free monoid on one generator). This in turn allows us to establish the homotopy commutativity of diagrams 11.10 and 11.11. In addition, a similar sort of use of the $M=3$ case of the Pre-Theorem yields the homotopy commutativity of diagram 11.8 .

We emphasize again that we do not yet know how decisive the Pre-Theorem will be in making progress on our more ambitious program. It does seem clear, though, that these issues are closely related to more subtle centrality properties of $v_{n}$ self-maps.

\section{Appendix 3: The category of $S$-modules}

We begin by providing an outline of the construction of the category of $S$-modules and its smash product. Our main reference is, of course [5], although the reader might also wish to look at [7] for background information on spectra and prespectra.

One starts with a universe $U$, an inner product space isomorphic to $\mathbb{R}^{\infty}$, topologized as the colimit of its finite dimensional subspaces. An indexing set $\mathscr{A}$ in $U$ is a set of finite dimensional vector subspaces of $U$, cofinal in the set of all finite dimensional subspaces of $U$ ordered by inclusion. If $V$ is a finite dimensional subspace of $U$, let $S(V)$ denote the one-point compactification of $V$ (with base point the point at $\infty$ ) as in Sect. 3, and for $X$ a pointed space, let $\Sigma^{V} X=S(V) \wedge X$. A prespectrum $E$ indexed on $\mathscr{A}$ consists of a pointed space $E V$ for each $V \in \mathscr{A}$, together with based maps $\sigma: \Sigma^{W-V} E V \rightarrow E W$ for $V \subset W$ in $\mathscr{A}$ such that $\sigma$ is the identity when $W=V$ and such that the diagram

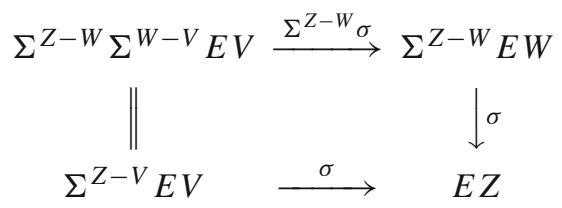

commutes whenever $V \subset W \subset Z$. Here we define $W-V$ to be the orthogonal complement of $V$ in $W$ whenever $V \subset W$. Maps between prespectra indexed on $\mathscr{A}$ are defined in the evident way, and we denote this category of prespectra by $\mathscr{P} \mathscr{A}(U)$. A prespectrum $E$ is a spectrum if each of the adjoint maps $\tilde{\sigma}: E V \rightarrow \Omega^{W-V} E W$ are homeomorphisms. This category of spectra is denoted $\mathscr{S} \mathscr{A}(U)$. The forgetful functor $\mathscr{S} \mathscr{A}(U) \rightarrow \mathscr{P} \mathscr{A}(U)$ has a left adjoint $L: \mathscr{P} \mathscr{A}(U) \rightarrow \mathscr{S} \mathscr{A}(U)$ referred to as spectrification; this implies that $\mathscr{S} \mathscr{A}(U)$ has colimits - the colimit of a diagram 
in $\mathscr{S} \mathscr{A}(U)$ is just $L$ applied to the colimit of the diagram regarded as a diagram in $\mathscr{P} \mathscr{A}(U)$. In addition, $L$ preserves colimits.

If $X$ is a pointed space, there is a functor $X \wedge ?: \mathscr{P} \mathscr{A}(U) \rightarrow \mathscr{P} \mathscr{A}(U)$ given in the usual way: $(X \wedge E)(V)=X \wedge E V$. By applying the spectrification functor $L$ to $X \wedge E$, we obtain a functor from $\mathscr{S} \mathscr{A}(U)$ to $\mathscr{S} \mathscr{A}(U)$ which we also denote $X \wedge$ ?. Moreover, if $E$ is a prespectrum, $L(X \wedge E) \cong L(X \wedge L E)$, so that $L$ preserves smash products with pointed spaces. If a functor between categories of (pre)spectra preserves smash products with pointed spaces as well as colimits, we will say that the functor preserves all colimits.

Although indexing sets are necessary for dealing with prespectra-for example, the external smash product of two prespectra is not defined on all finite dimensional subspaces of its universe of definition- $\mathscr{S} \mathscr{A}(U)$ is canonically isomorphic to $\mathscr{S}(U)$, where the indexing set in this latter category of spectra is taken to be the set of all finite dimensional subspaces of $U$. For this reason, we will omit references to indexing sets and work with $\mathscr{S}(U)$ and $\mathscr{P}(U)$, although some constructions with prespectra require specific choices of indexing sets. If $U=\mathbb{R}^{\infty}$, the universe we choose when dealing with $S$-modules, we will write $\mathscr{S}$ and $\mathscr{P}$ for $\mathscr{S}(U)$ and $\mathscr{P}(U)$ respectively. We also remark that, in this situation, the value of a spectrum on the subspace $\mathbb{R}^{n}$ is the " $n$th space" of the spectrum.

If $E \in \mathscr{P}(U)$ and $E^{\prime} \in \mathscr{P}\left(U^{\prime}\right)$, the external smash product $E \bar{\wedge} E^{\prime} \in \mathscr{P}\left(U \oplus U^{\prime}\right)$ is defined by

$$
\left(E \bar{\wedge} E^{\prime}\right)\left(V \oplus V^{\prime}\right)=E V \wedge E^{\prime} V^{\prime}
$$

(Our notation $\bar{\wedge}$ may not be entirely standard; we are using it to distinguish the external from the internal smash product.) If $E$ and $E^{\prime}$ are spectra, we may define a spectrum $E \bar{\wedge} E^{\prime}$ by applying $L$ to the prespectrum $E \bar{\wedge} E^{\prime}$. The external smash product preserves all colimits and behaves well with respect to spectrification; i.e., $L E \bar{\wedge} L E^{\prime} \cong L\left(E \bar{\wedge} E^{\prime}\right)$ for any prespectra $E$ and $E^{\prime}$.

The construction of internal smash products requires the notion of twisted halfsmash products to go from spectra in $\mathscr{S}(U \oplus U)$ to spectra in $\mathscr{S}(U)$. We follow "Appendix A" of [5], due to Michael Cole, in our treatment. If $U$ and $U^{\prime}$ are universes, an object $\mathscr{E}$ of the category $\mathscr{S}\left(U^{\prime} ; U\right)$ consists of a spectrum $\mathscr{E} V \in \mathscr{S}\left(U^{\prime}\right)$ for each finite dimensional subspace $V$ of $U$, together with isomorphisms $\Sigma^{W-V} \mathscr{E}_{W} \rightarrow$ $\mathscr{E}_{V}$ whenever $V \subset W$. These isomorphisms must satisfy the evident compatibility condition when $V \subset W \subset Z$. Now let $D$ be in $\mathscr{P}(U)$. For finite dimensional subspaces $V, W$ of $U$ with $V \subset W$, we have the map

$$
\mathscr{E}_{V} \wedge D V \cong \Sigma^{W-V} \mathscr{E}_{W} \wedge D V \longrightarrow \mathscr{E}_{W} \wedge D W
$$

of spectra. We then define $\mathscr{E} \wedge D \in \mathscr{S}\left(U^{\prime}\right)$ by

$$
\mathscr{E} \wedge D=\operatorname{colim}_{V \subset U}(\mathscr{E} \wedge D V)
$$

The functor $\mathscr{E} \wedge ?: \mathscr{P}(U) \rightarrow \mathscr{S}\left(U^{\prime}\right)$ preserves all colimits and moreover, $\mathscr{E} \wedge L D \cong$ $\mathscr{E} \wedge D$. 
Let $\mathscr{I}\left(U, U^{\prime}\right)$ denote the space of isometries from $U$ to $U^{\prime}$; that is, an element of $\mathscr{I}\left(U, U^{\prime}\right)$ is an inner product preserving linear transformation from $U$ to $U^{\prime}$. Let $\alpha: A \rightarrow \mathscr{I}\left(U, U^{\prime}\right)$ be a continuous map. In [5, "Appendix A"], an object $\mathscr{M} \alpha \in$ $\mathscr{S}\left(U^{\prime} ; U\right)$ is constructed using Thom spaces of various vector bundles over subspaces of $A$, and the twisted half-smash product $\alpha \ltimes ?: \mathscr{S}(U) \rightarrow \mathscr{S}\left(U^{\prime}\right)$ is defined by

$$
\alpha \ltimes E=\mathscr{M} \alpha \wedge E .
$$

If the map $\alpha$ is understood, we will often write $\alpha \ltimes E$ as $A \ltimes E$, and if $A=*$ with $\alpha(*)=f \in \mathscr{I}\left(U, U^{\prime}\right)$, we often write $f_{*} E$ for $\alpha \ltimes E$. (We remark here that we also use the notation $A \ltimes E$ for $A_{+} \wedge E$ when $A$ is just a space and there is no "change of universe" going on. This is indeed the case elsewhere throughout the paper: we only use the twisted half-smash product here in passing from external to internal smash products).

The twisted half-smash product has some additional properties of importance. If $\alpha$ is as above and $\beta: B \rightarrow \mathscr{I}\left(U^{\prime}, U^{\prime \prime}\right)$, let $\beta \times_{c} \alpha$ denote the composition

$$
B \times A \longrightarrow \mathscr{I}\left(U^{\prime}, U^{\prime \prime}\right) \times \mathscr{I}\left(U, U^{\prime}\right) \stackrel{\circ}{\longrightarrow} \mathscr{I}\left(U, U^{\prime \prime}\right),
$$

where o denotes the usual composition pairing. If $\gamma: C \rightarrow \mathscr{I}\left(U_{1}, U_{1}^{\prime}\right)$ and $\delta: D \rightarrow$ $\mathscr{I}\left(U_{2}, U_{2}^{\prime}\right)$, let $\gamma \times_{\oplus} \delta$ denote the composition

$$
C \times D \longrightarrow \mathscr{I}\left(U_{1}, U_{1}^{\prime}\right) \times \mathscr{I}\left(U_{2}, U_{2}^{\prime}\right) \stackrel{\oplus}{\longrightarrow} \mathscr{I}\left(U_{1} \oplus U_{2}, U_{1}^{\prime} \oplus U_{2}^{\prime}\right)
$$

where $\oplus$ denotes the evident map. We then have natural isomorphisms

$$
\left(\beta \times_{c} \alpha\right) \ltimes E \cong \beta \ltimes(\alpha \ltimes E)
$$

and

$$
\left(\gamma \times_{\oplus} \delta\right) \ltimes\left(E_{1} \bar{\wedge} E_{2}\right) \cong\left(\gamma \ltimes E_{1}\right) \bar{\wedge}\left(\delta \ltimes E_{2}\right) .
$$

This latter isomorphism follows immediately from a canonical isomorphism

$$
\mathscr{M} \gamma \bar{\wedge} \delta \cong \mathscr{M}\left(\gamma \times_{\oplus} \delta\right)
$$

where $\mathscr{M} \gamma \bar{\wedge} \mathscr{M} \delta \in \mathscr{S}\left(U_{1}^{\prime} \oplus U_{2}^{\prime} ; U_{1} \oplus U_{2}\right)$ is given by

$$
(\mathscr{M} \gamma \bar{\wedge} \mathscr{M})_{\left(V_{1} \oplus V_{2}\right)}=(\mathscr{M} \gamma)_{V_{1}} \bar{\wedge}(\mathscr{M} \delta)_{V_{2}}
$$

Now fix a universe $U$, and let $U^{j}$ be the $j$-fold direct sum of $U$. Let $\mathscr{L}(j)=$ $\mathscr{I}\left(U^{j}, U\right)$; these spaces form an operad called the linear isometries operad. There is a monad $\mathbb{L}$ in $\mathscr{S} U$ with $\mathbb{L} E=\mathscr{L}(1) \ltimes E$, where $\mathscr{L}(1) \rightarrow \mathscr{I}(U, U)$ is the identity, and the category $\mathbb{L}[\mathscr{S} U]$ of $\mathbb{L}$-algebras in $\mathscr{S} U$ will also be called the category of $\mathbb{L}$-spectra. For later use, we remark here that $\Sigma^{\infty} X$ is canonically an $\mathbb{L}$-spectrum for any pointed space $X$. 
If $M$ and $N$ are in $\mathbb{L}[\mathscr{S} U], M \wedge \mathscr{L} N$ is defined as the coequalizer of the two maps

$$
(\mathscr{L}(2) \times \mathscr{L}(1) \times \mathscr{L}(1)) \ltimes(M \bar{\wedge} N) \Longrightarrow \mathscr{L}(2) \ltimes(M \bar{\wedge} N),
$$

where the top map is induced by the operad structure map $\mathscr{L}(2) \times \mathscr{L}(1) \times \mathscr{L}(1) \rightarrow$ $\mathscr{L}(2)$, and the bottom map is the composition

$$
\begin{aligned}
(\mathscr{L}(2) \times \mathscr{L}(1) \times \mathscr{L}(1)) \ltimes(M \bar{\wedge}) & \cong \mathscr{L}(2) \ltimes[(\mathscr{L}(1) \times \mathscr{L}(1)) \ltimes(M \bar{\wedge} N)] \\
& \cong \mathscr{L}(2) \ltimes(\mathbb{L} M \bar{\wedge} N) \\
& \rightarrow \mathscr{L}(2) \ltimes(M \wedge N) .
\end{aligned}
$$

The last map in this composition is induced by the $\mathbb{L}$-algebra structure maps $\mathbb{L} M \rightarrow$ $M$ and $\mathbb{L} N \rightarrow N$. The pairing $\wedge \mathscr{L}$ is easily seen to be commutative. Indeed, let $t \in \mathscr{I}\left(U^{2}, U^{2}\right)$ denote the isometry sending $(v, w)$ to $(w, v)$; the switch map $M V \wedge$ $N V^{\prime} \rightarrow N V^{\prime} \wedge M V$ then induces an isomorphism $M \bar{\wedge} N \rightarrow t_{*}(N \bar{\wedge} M)$. This implies that

$$
\mathscr{L}(2) \ltimes(M \bar{\wedge} N) \stackrel{\cong}{\longrightarrow} \mathscr{L}(2) \ltimes t_{*}(N \bar{\wedge} M) \cong \alpha \ltimes(N \bar{\wedge} M),
$$

where $\alpha: \mathscr{L}(2) \rightarrow \mathscr{I}\left(U^{2}, U^{2}\right)$ is given by $\alpha(f)=f t$. But composition by $t$ yields an isomorphism from $\alpha$ to the identity map over $\mathscr{I}\left(U^{2}, U\right)$; hence

$$
\alpha \ltimes(N \bar{\wedge} M) \stackrel{\cong}{\longrightarrow} \mathscr{L}(2) \ltimes(N \bar{\wedge} M) .
$$

Similarly, there is an isomorphism

$$
(\mathscr{L}(2) \times \mathscr{L}(1) \times \mathscr{L}(1)) \ltimes(M \bar{\wedge} N) \stackrel{\cong}{\longrightarrow}(\mathscr{L}(2) \times \mathscr{L}(1) \times \mathscr{L}(1)) \ltimes(N \bar{\wedge} M)
$$

which yields a map of coequalizer diagrams and hence an isomorphism

$$
\tau: M \wedge \mathscr{L} N \stackrel{\cong}{\longrightarrow} N \wedge \mathscr{L} M
$$

Using further properties of the linear isometries operad, one can prove that $\wedge \mathscr{L}$ is also associative. It is however not quite unital. There is a natural map $\lambda: \Sigma^{\infty} S^{0} \wedge \mathscr{L} M \rightarrow$ $M$ of $\mathbb{L}$-spectra ([5, I, Proposition 8.3]) which is always a weak equivalence ([5, I, Theorem 8.5]), but not an isomorphism in general. If $M=\Sigma^{\infty} S^{0}$ —or more generally any suspension spectrum $-\lambda$ is an isomorphism; it then follows (using commutativity and associativity properties of $\lambda$ ) that

$$
\lambda: \Sigma^{\infty} S^{0} \wedge \mathscr{L}\left(\Sigma^{\infty} S^{0} \wedge \mathscr{L} M\right) \longrightarrow \Sigma^{\infty} S^{0} \wedge \mathscr{L} M
$$

is an isomorphism for any $\mathbb{L}$-spectrum $M$. 
The category $\mathscr{M}$ of $S$-modules is defined to be the full subcategory of $\mathbb{L}[\mathscr{S}]$ consisting of those objects for which $\lambda$ is an isomorphism. If $M$ and $N$ are $S$-modules, then $M \wedge \mathscr{L} N$ is an $S$-module, and we define

$$
M \wedge N \equiv M \wedge_{S} N \equiv M \wedge \mathscr{L} N
$$

With this smash product, the category of $S$-modules becomes symmetric monodical. The categories $\mathscr{S}, \mathbb{L}[\mathscr{S}]$, and $\mathscr{M}$ are all model categories over pointed topological spaces - the weak equivalences in all three are the maps inducing isomorphisms on stable homotopy groups of spectra-and the functors $\mathbb{L}(?): \mathscr{S} \rightarrow \mathbb{L}[\mathscr{S}]$ and $\Sigma^{\infty} S^{0} \wedge \mathscr{L} ?: \mathbb{L}[\mathscr{S}] \rightarrow \mathscr{M}$ are both left adjoints of Quillen equivalences.

Despite the point-set technicalities involved in the construction of $S$-modules, these objects can often-without undue effort—-be dealt with as flexibly as ordinary topological spaces. As an example, we provide here a detailed proof of Proposition 5.1; the proofs of Propositions 5.3 and 5.4 are similar.

To prove Proposition 5.1, start by considering the cofiber $C(f \bar{\wedge} X, X \bar{\wedge} f)$ of the map

$$
(f \bar{\wedge} X, X \bar{\wedge} f): \Sigma^{k} X \bar{\wedge} X \vee X \bar{\wedge} \Sigma^{k} X \longrightarrow X \bar{\wedge} X
$$

We have a natural isomorphism

$$
\zeta_{f}: C(f \bar{\wedge} X, X \bar{\wedge} f) \longrightarrow C(f) \bar{\wedge} X \cup X \bar{\wedge} C(f)
$$

in $\mathscr{S}\left(\mathbb{R}^{\infty} \oplus \mathbb{R}^{\infty}\right)$; this is proved by observing that it holds at the level of prespectra (by the usual argument for pointed spaces) and then using the fact that spectrification commutes with all colimits as well as with the external smash product of prespectra. The space level equivariance of the above isomorphism implies that $\zeta_{f}$ is equivariant in the following sense. The diagram

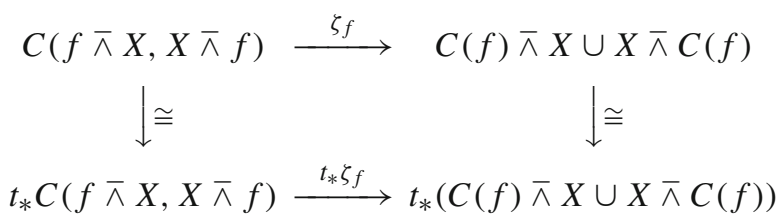

is commutative, where the vertical maps are the isomorphisms induced by the switch maps. This then implies that the isomorphism

$\mathscr{L}(2) \ltimes C(f \bar{\wedge} X, X \bar{\wedge} f) \stackrel{\mathscr{L}(2) \ltimes \zeta_{f}}{\longrightarrow} \mathscr{L}(2) \ltimes(C(f) \wedge X \cup X \bar{\wedge} C(f))$

is equivariant.

Now the isomorphism $\zeta_{f}$ also behaves well with respect to smash products with pointed spaces; together with 12.1 and the definition of twisted half-smash products, 
this yields a commutative diagram

$$
\begin{aligned}
& (\mathscr{L}(1) \times \mathscr{L}(1)) \ltimes C(f \bar{\wedge} X, X \bar{\wedge} f) \stackrel{\cong}{\longrightarrow} C(\mathbb{L} f \bar{\wedge} X, \mathbb{L} X \bar{\wedge} f) \\
& \downarrow(\mathscr{L}(1) \times \mathscr{L}(1)) \ltimes \zeta_{f} \\
& (\mathscr{L}(1) \times \mathscr{L}(1)) \ltimes(C(f) \bar{\wedge} X \cup X \bar{\wedge} C(f)) \stackrel{\cong}{\longrightarrow} C(\mathbb{L} f) \bar{\wedge} X \cup \mathbb{L} X \bar{\wedge} C(\mathbb{L} f) .
\end{aligned}
$$

Since $f$ is a map of $\mathbb{L}$-spectra, there is also a commutative diagram

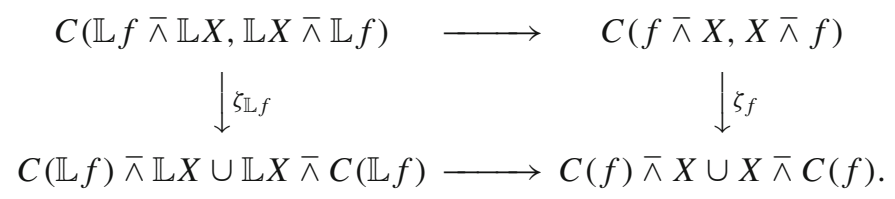

Finally, there are diagrams

$$
(\mathscr{L}(2) \times \mathscr{L}(1) \times \mathscr{L}(1)) \ltimes C(f \wedge X, X \bar{\wedge} f) \Longrightarrow \mathscr{L}(2) \ltimes C(f \bar{\wedge} X, X \bar{\wedge} f)
$$

and

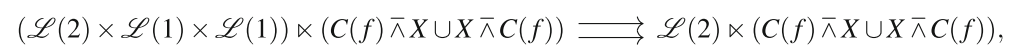

whose coequalizers are $C(f \wedge X, X \wedge f)$ and $C(f) \wedge X \cup X \wedge C(f)$ respectively. Each top map in these diagrams comes from the action of $\mathscr{L}(1) \times \mathscr{L}(1)$ on $\mathscr{L}(2)$, and each bottom map comes from the $\mathbb{L} \times \mathbb{L}$-algebra structure on the respective spectra. By 12.2 and 12.3, the isomorphisms $(\mathscr{L}(2) \times \mathscr{L}(1) \times \mathscr{L}(1)) \ltimes \zeta_{f}$ and $\mathscr{L}(2) \ltimes \zeta_{f}$ give an isomorphism between the above diagrams and hence a natural isomorphism from $C(f \wedge X, X \wedge f)$ to $C(f) \wedge X \cup X \wedge C(f)$. Since $\mathscr{L}(2) \ltimes \zeta_{f}$ is equivariant, so is this isomorphism.

\section{References}

1. Bruner, R.R., May, J.P., McClure, J.E., Steinberger, M.: $H_{\infty}$ ring spectra and their applications. Lecture Notes in Math, vol. 1176. Springer, Berlin (1986)

2. Devinatz, E.S.: Small ring spectra. J. Pure Appl Algebra 81, 11-16 (1992)

3. Dwyer, W.G., Kan, D.M., Smith, J.H.: Homotopy commutative diagrams and their realizations. J Pure Appl Algebra 57, 5-24 (1989)

4. Elmendorf, A.D.: Stabilization as a CW approximation. J Pure Appl Algebra 140, 23-32 (1999)

5. Elmendorf, A.D., Kriz, I., Mandell, M.A., May, J.P.: Rings, modules, and algebras in stable homotopy theory. Surveys and Monographs, vol. 47. American Mathematical Society, Providence (1997)

6. Hopkins, M.J., Smith, J.H.: Nilpotence and stable homotopy theory II. Ann. Math. 138, 1-49 (1998)

7. Lewis Jr, L.G., May, J.P., Steinberger, M.: Equivariant stable homotopy theory (with contributions by J.E. McClure), Lecture Notes in Math. vol. 1213. Springer, Berlin (1986)

8. May, J.P.: The geometry of iterated loop spaces. Lecture Notes in Math, vol. 271. Springer, Berlin (1972)

9. Nishida, G.: The nilpotency of elements of the stable homotopy groups of spheres. J. Math. Soc. Jpn. 25, 707-732 (1973)

10. Oka, S.: Ring spectra with few calls. Jpn J. Math. 5, 81-100 (1979) 
11. Oka, S.: Multiplicative structure of finite ring spectra and stable homotopy of spheres. In: Madsen, I., Oliver, B. (eds.) Algebraic topology. Aarhus 1982, Lecture Notes in Math, vol. 1051, pp. 418-441. Springer, Berlin (1984)

12. Ravenel, D.C.: Localization with respect to certain periodic homology theories. Am. J. Math. 106, 415-446 (1984)

13. Steenrod, N.E.: A convenient category of topological spaces. Michigan Math. J. 14, 133-152 (1967) 